\title{
Bayesian Mixture Modeling for Multivariate Conditional Distributions
}

\author{
Maria De Yoreo and Jerome P. Reiter*
}

\begin{abstract}
We present a Bayesian mixture model for estimating the joint distribution of mixed ordinal, nominal, and continuous data conditional on a set of fixed variables. The model uses multivariate normal and categorical mixture kernels for the random variables. It induces dependence between the random and fixed variables through the means of the multivariate normal mixture kernels and via a truncated local Dirichlet process. The latter encourages observations with similar values of the fixed variables to share mixture components. Using a simulation of data fusion, we illustrate that the model can estimate underlying relationships in the data and the distributions of the missing values more accurately than a mixture model applied to the random and fixed variables jointly. We use the model to analyze consumers' reading behaviors using a quota sample, i.e., a sample where the empirical distribution of some variables is fixed by design and so should not be modeled as random, conducted by the book publisher HarperCollins.
\end{abstract}

Key words: Dirichlet process, fusion, imputation, missing, mutual information.

\section{Introduction}

Bayesian mixture models are flexible and convenient tools for estimating the joint distribution of a set of variables (e.g., Dunson and Xing, 2009; Banerjee et al., 2013; Müller and Mitra,

*M. DeYoreo (maria.deyoreo@stat.duke.edu) is postdoctoral researcher and J. Reiter is Mrs. Alexander Hehmeyer Professor of Statistics, Department of Statistical Science. This research was supported in part by The National Science Foundation under award SES-11-31897. 
2013). Often, however, it is desirable to treat some of the variables as conditioning information rather than random variables. For example, when data are collected using a stratified or quota sampling design, the empirical distribution of the design variables is fixed a priori. On principle, it does not make sense to estimate their distribution with uncertainty. In fact, when the sampling is not proportional to population shares, treating the design variables as random can result in badly biased estimates of population-level quantities (Schifeling and Reiter, 2016; Kunihama et al., 2016; Fosdick et al., 2016). As another example, when using mixture models for multiple imputation of missing data (Rubin, 1987), it is unnecessary to estimate the marginal distribution of the variables with no missing values. Rather, all we need is the conditional distribution of variables with missingness given those that are fully observed.

In this article, we present mixture models for estimating the joint distribution of variables treated as random conditional on a set of variables treated as fixed. The models use multivariate normal kernels for continuous and ordinal variables (via a probit specification), and independent multinomial kernels for nominal variables. We induce dependence between the random and fixed variables in two ways. First, for continuous and ordinal variables, we let the mean of the multivariate normal distribution within each mixture component be a function of the fixed variables. Second, we encourage observations that have similar values of the fixed variables to share mixture components via a local Dirichlet process (Chung and Dunson, 2011). The local Dirichlet process facilitates estimation of the dependence between the nominal random variables and the fixed variables, which is otherwise difficult to capture. It also offers the model additional flexibility to capture relationships between the random continuous/ordinal variables and the fixed variables.

The proposed conditional mixture model can have advantages over other approaches commonly used for estimating multivariate conditional distributions of mixed data. For example, one alternative is to eschew mixtures altogether and specify multivariate linear or logistic regressions. Such models make strong assumptions, e.g., linearity and Gaussian errors, and can require challenging model specification tasks, e.g., selecting which interaction effects to 
include in logistic regressions. By comparison, mixture models tend to be more capable at capturing complex distributional features (Müller and Quintana, 2004; Norets and Pelenis, 2012, Papageorgiou et al., 2015). Another alternative is to treat all variables as random, estimate their joint distribution via a mixture model, and derive relevant conditional distributions from the resulting estimates (Müller et al., 1996; Shahbaba and Neal, 2009, Dunson and Bhattacharya, 2010; Hannah et al., 2011). Even with (modest-sized) representative samples, a full mixture model can waste fitting power on the joint distribution of the fixed variables, as it seeks to fit the entire joint distribution. This can result in poor predictive inference for conditional distributions (Wade et al. 2014). A third alternative is to use a mixture model in which the mixture weights depend on the fixed variables (e.g., Griffin and Steel, 2006; Dunson and Park, 2008). However, it can be complicated to estimate and obtain inferences from such models, particularly when the number of fixed variables is not small.

The remainder of this article is organized as follows. In Section 2 , we describe the mixture model for multivariate conditional inference with mixed data, which we refer to as CMM-Mix. We also describe a variable selection procedure based on estimated mutual information values (Battiti, 1994; Ding and Peng, 2003; Eztévez et al., 2009) that can be used to trim variables from the conditioning set that determines the local weights, which can be useful when the set contains many variables. In Section 3, we compare CMM-Mix to a full mixture model in a simulation study of techniques for data fusion (Rässler, 2004; Gilula et al., 2006; D'Orazio et al. 2006), which is a type of missing data scenario common in marketing contexts. In Section 4, we analyze data from a quota sample from HarperCollins Publishers, in which we seek to understand relationships involving individuals' reading behaviors and interests; for example, what distinguishes people who own eBooks from those who do not? In Section5, we conclude with future directions for research. This article is accompanied by supplementary material that presents additional results from simulations and data illustrations. 


\section{Methodology}

Suppose that the collected data include $p$ variables that the analyst treats as random, and $q$ variables that the analyst treats as fixed. For $i=1, \ldots, n$, let $Y_{i j}^{(R)} \in\left\{1, \ldots, k_{j}^{(R)}\right\}$ be the value of ordinal random variable $j$ for individual $i$, for $j=1, \ldots, p_{o}$; let $X_{i j}^{(R)} \in\left\{1, \ldots, d_{j}^{(R)}\right\}$ be the value of nominal random variable $j$ for individual $i$, for $j=1, \ldots, p_{n}$; and, let $Z_{i j}^{(R)}$ be the standardized value of continuous random variable $j$ for individual $i$, for $j=1, \ldots, p_{c}$. To facilitate modeling, we introduce a latent continuous random variable $W_{i j}^{(R)}$ for each $Y_{i j}^{(R)}$. Similarly, for each $i$ let there be $q_{o}$ ordinal fixed variables $Y_{i j}^{(F)} \in\left\{1, \ldots, k_{j}^{(F)}\right\}, q_{n}$ nominal fixed variables $X_{i j}^{(F)} \in\left\{1, \ldots, d_{j}^{(F)}\right\}$, and $q_{c}$ standardized continuous fixed variables $Z_{i j}^{(F)}$. We write each individual's data as the vector $\left(\mathbf{Y}_{i}^{(R)}, \mathbf{Z}_{i}^{(R)}, \mathbf{X}_{i}^{(R)}, \mathbf{Y}_{i}^{(F)}, \mathbf{Z}_{i}^{(F)}, \mathbf{X}_{i}^{(F)}\right)$. Writing the variables generically, we seek to construct a mixture model for $p\left(\mathbf{Y}^{(R)}, \mathbf{Z}^{(R)}, \mathbf{X}^{(R)} \mid\right.$ $\left.\mathbf{Y}^{(F)}, \mathbf{Z}^{(F)}, \mathbf{X}^{(F)}\right)$. To simplify notation, we sometimes refer to the set $\left(\mathbf{Y}^{(R)}, \mathbf{Z}^{(R)}, \mathbf{X}^{(R)}\right)$ as $\mathcal{R}$ and the set $\left(\mathbf{Y}^{(F)}, \mathbf{Z}^{(F)}, \mathbf{X}^{(F)}\right)$ as $\mathcal{F}$.

\subsection{Modeling strategy: Connecting $\mathcal{R}$ and $\mathcal{F}$ via CMM-Mix}

Let $H_{i} \in\{1, \ldots, N\}$ be a mixture allocation variable representing the component observation $i$ belongs to, such that $H_{i}=l$ if and only if observation $i$ belongs to component $l$. At the level of the data, for any individual $i$ the model for CMM-Mix is

$$
\begin{aligned}
\left(\mathbf{W}_{i}^{(R)}, \mathbf{Z}_{i}^{(R)} \mid\left\{\boldsymbol{\beta}_{h}\right\},\left\{\boldsymbol{\Sigma}_{h}\right\}, H_{i}, \mathbf{X}_{i}^{(R)}, \mathcal{F}\right) & \sim \mathrm{N}\left(\boldsymbol{D}\left(\mathbf{X}_{i}^{(R)}, \mathbf{Y}_{i}^{(F)}, \mathbf{Z}_{i}^{(F)}, \mathbf{X}_{i}^{(F)}\right) \boldsymbol{\beta}_{H_{i}}, \boldsymbol{\Sigma}_{H_{i}}\right) \\
\left(\mathbf{X}_{i}^{(R)} \mid\left\{\boldsymbol{\psi}_{h}\right\}, H_{i}\right) & \sim \prod_{j=1}^{p_{n}} \operatorname{categ}\left(\psi_{H_{i}, 1}^{(j)}, \ldots, \psi_{H_{i}, d_{j}^{(R)}}^{(j)}\right) .
\end{aligned}
$$

Here, $\boldsymbol{D}(\cdot)$ is a design vector of length $k$ encoding main effects and possibly non-linear terms identified through exploratory data analysis as helpful for capturing local dependence, and $\boldsymbol{\beta}_{h}$ is a $k \times\left(p_{c}+p_{o}\right)$ matrix of regression coefficients. The ordinal $\mathbf{Y}^{(R)}$ are determined from $\mathbf{W}^{(R)}$ such that $Y_{i j}^{(R)}=l$ if and only if $\gamma_{j, l-1}<W_{i j}^{(R)} \leq \gamma_{j, l}$, for $l=1, \ldots, k_{j}^{(R)}$, and $-\infty=\gamma_{j, 0}<\gamma_{j, 1}<\cdots<\gamma_{j, k_{j}^{(R)}-1}<\gamma_{j, k_{j}^{(R)}}=\infty$.

The mixture of regressions helps capture dependence between $\left(\mathbf{Y}^{(R)}, \mathbf{Z}^{(R)}\right)$ and $\mathcal{F}$. It also strengthens the connections between $\left(\mathbf{Y}^{(R)}, \mathbf{Z}^{(R)}\right)$ and $\mathbf{X}^{(R)}$ by allowing for local dependence 
within components. This regression approach is related to the ANOVA dependent Dirichlet process (DP) model of De Iorio et al. (2004) and also is used by Murray and Reiter (2016) in a full mixture model. We explicitly avoid the regression approach for connecting $\mathbf{X}^{(R)}$ and $\mathcal{F}$, primarily because it can be computationally challenging to implement with MCMC sampling. For example, multinomial logistic regressions for $\mathbf{X}^{(R)}$ within components can introduce a large number of regression parameters for which there are no conjugate priors.

With only (1) and (2), the model has to capture dependence between $\mathbf{X}^{(R)}$ and $\mathcal{F}$ through a convoluted path involving the regressions for $\left(\mathbf{Y}^{(R)}, \mathbf{Z}^{(R)}\right)$. With modest sample sizes, this path seems unlikely to be up to the task. We therefore strengthen the connections using a truncated local Dirichlet process (LDP). This provides a prior distribution for a collection of random distributions indexed by fixed variables $\mathcal{F}$, in which units that have similar values of $\mathcal{F}$ are assumed to share similar distributions for $\mathcal{R}$. The dependence between distributions associated with fixed variables $\boldsymbol{f}$ and $\boldsymbol{f}^{\prime}$ increases as the distance between $\boldsymbol{f}$ and $\boldsymbol{f}^{\prime}$ decreases. This prior possesses many attractive properties, such as retaining a marginal DP prior for any $\boldsymbol{f}$, as described by Chung and Dunson (2011).

Each $H_{i}$ arises from a subset of $\{1, \ldots, N\}$, where the particular subset is chosen according to the value of $\mathcal{F}$ for observation $i$. The truncated LDP is built from sequences of mutually independent random variables: $\left\{V_{h}: h=1,2, \ldots, N\right\}$ are beta $(1, \alpha)$ distributed random variables that determine the stick-breaking weights, $\left\{\boldsymbol{\theta}_{h}=\left(\boldsymbol{\beta}_{h}, \boldsymbol{\Sigma}_{h}, \boldsymbol{\psi}_{h}\right): h=1,2, \ldots, N\right\}$ are the atoms, and $\left\{\boldsymbol{\Gamma}_{h}: h=1,2, \ldots, N\right\}$ are locations in $\mathcal{S}$, the sample space of $\mathcal{F}$. The mixture weights and probabilities associated with an observation having a particular $\mathcal{F}=\boldsymbol{f}$ are determined by the set of $\boldsymbol{\Gamma}_{h}$ that are in some neighborhood of $\boldsymbol{f}$. Specifically, let $\eta_{\boldsymbol{f}}=$ $\left\{h: d\left(\boldsymbol{f}, \boldsymbol{\Gamma}_{h}\right) \leq d^{*}\right\}$ be a set indexing the locations belonging to the $d^{*}$-neighborhood of $\boldsymbol{f}$. Here $d$ is a distance measure and $d^{*}$ represents the neighborhood size. Elements $\eta_{\boldsymbol{f}}$ of $\left\{V_{h}\right\}$ and $\left\{\boldsymbol{\theta}_{h}\right\}$ are then used in constructing $G_{\boldsymbol{f}}$, the random distribution associated with $\boldsymbol{f}$. The 
prior on the mixture components and weights is therefore:

$$
\begin{array}{r}
H_{i} \mid \boldsymbol{V}, \boldsymbol{\Gamma}, \mathcal{F}_{i}=\boldsymbol{f}_{i} \stackrel{\text { ind. }}{\sim} \sum_{h=1}^{N\left(\boldsymbol{f}_{i}\right)} p_{h}\left(\boldsymbol{f}_{i}\right) \delta_{\pi_{h}\left(\boldsymbol{f}_{i}\right)}(\cdot), i=1, \ldots, n \\
V_{h} \mid \alpha \stackrel{i . i . d .}{\sim} \operatorname{beta}(1, \alpha), h=1, \ldots, N \\
\boldsymbol{\Gamma}_{h} \stackrel{\text { i.i.d. }}{\sim} p\left(\boldsymbol{\Gamma}_{h}\right), h=1, \ldots, N
\end{array}
$$

where $p_{h}\left(\boldsymbol{f}_{i}\right)=V_{\pi_{h}\left(\boldsymbol{f}_{i}\right)} \prod_{j<h}\left(1-V_{\pi_{j}\left(\boldsymbol{f}_{i}\right)}\right)$ for $h=1, \ldots, N\left(\boldsymbol{f}_{i}\right)-1, \pi_{h}\left(\boldsymbol{f}_{i}\right)$ is the $h$ th ordered index in $\eta_{\boldsymbol{f}_{i}}$, and $N\left(\boldsymbol{f}_{i}\right)=\left|\eta_{\boldsymbol{f}_{i}}\right|$. The last element in each probability vector $p_{N\left(\boldsymbol{f}_{i}\right)}\left(\boldsymbol{f}_{i}\right)$ is determined so that $\sum_{h=1}^{N\left(\boldsymbol{f}_{i}\right)} p_{h}\left(\boldsymbol{f}_{i}\right)=1$. Although a global truncation level of $N$ is fixed, $N\left(\boldsymbol{f}_{i}\right)$ is still a random quantity. Thus, the number of components in each mixture distribution is data driven. For $p\left(\boldsymbol{\Gamma}_{h}\right)$, we use a product of independent uniforms for each variable in $\mathcal{F}$. We discuss the choice of distance function in Section 2.3.

The base distributions from which the atoms $\boldsymbol{\theta}_{h}$ are drawn from are given by:

$$
\begin{array}{r}
\boldsymbol{\beta}_{h} \stackrel{i . i . d .}{\sim} \operatorname{MN}_{k \times\left(p_{o}+p_{c}\right)}\left(\boldsymbol{\beta}_{0}, \operatorname{diag}\left(\tau_{1}^{2}, \ldots, \tau_{k}^{2}\right), \boldsymbol{I}_{p_{o}+p_{c}}\right), h=1, \ldots, N \\
\boldsymbol{\Sigma}_{h} \stackrel{i . i . d .}{\sim} \operatorname{IW}(\nu, \boldsymbol{S}), h=1, \ldots, N \\
\boldsymbol{\psi}_{h}^{(j)} \stackrel{i . i . d .}{\sim} \operatorname{Dirichlet}\left(a_{1}^{(j)}, \ldots, a_{d_{j}}^{(j)}\right), h=1, \ldots, N, j=1, \ldots, p_{n}
\end{array}
$$

where $\mathrm{MN}_{k \times\left(p_{o}+p_{c}\right)}$ denotes a matrix-normal distribution of dimension $k$ by $p_{o}+p_{c}$. This implies that $\operatorname{vec}\left(\boldsymbol{\beta}_{h}\right) \sim \mathrm{N}_{k\left(p_{o}+p_{c}\right)}\left(\operatorname{vec}\left(\boldsymbol{\beta}_{0}\right), \boldsymbol{I}_{p_{o}+p_{c}} \otimes \operatorname{diag}\left(\tau_{1}^{2}, \ldots, \tau_{k}^{2}\right)\right)$, where $\operatorname{vec}\left(\boldsymbol{\beta}_{h}\right)$ denotes the vectorization of $\boldsymbol{\beta}_{h}$, obtained by stacking its columns. The model is completed with hyperpriors on the parameters $\boldsymbol{\beta}_{0}, \boldsymbol{S}, \boldsymbol{\tau}$, and $\alpha$. Prior specification is discussed in Appendix A.1, including justification for the choice of the base distribution for $\boldsymbol{\beta}_{h}$.

\section{$2.2 \quad$ Model properties}

To describe the properties of CMM-Mix, it is useful first to marginalize over the mixture allocation indicators to obtain the multivariate conditional density for $\mathcal{R}$. We have

$$
\begin{aligned}
f\left(\mathbf{W}^{(R)}, \mathbf{Z}^{(R)}, \mathbf{X}^{(R)} \mid \mathcal{F}=\boldsymbol{f}\right)= \\
\quad \sum_{h=1}^{N(\mathcal{F})} p_{h}(\boldsymbol{f}) \mathrm{N}\left(\mathbf{W}^{(R)}, \mathbf{Z}^{(R)} ; \boldsymbol{D}\left(\mathbf{X}^{(R)}, \boldsymbol{f}\right) \boldsymbol{\beta}_{\pi_{h}(\boldsymbol{f})}, \boldsymbol{\Sigma}_{\pi_{h}(\boldsymbol{f})}\right) \prod_{j=1}^{p_{n}} \operatorname{categ}\left(X_{j}^{(R)} ; \boldsymbol{\psi}_{\pi_{h}(\boldsymbol{f})}^{(j)}\right) .
\end{aligned}
$$


We manipulate this expression to derive statements about the multivariate conditional distributions of the random variables at any $\boldsymbol{f}$.

Marginalizing (5) over $\mathbf{W}^{(R)}$ and $\mathbf{Z}^{(R)}$, we find that the marginal distribution for $\mathbf{X}^{(R)}$ is a mixture of independent multinomials. In particular, we have $\operatorname{Pr}\left(\mathbf{X}^{(R)}=\mathbf{x} \mid \mathcal{F}=\right.$ $\boldsymbol{f})=\sum_{l=1}^{N(\boldsymbol{f})} p_{l}(\boldsymbol{f}) \prod_{j=1}^{p_{n}} \psi_{\pi_{l}(\boldsymbol{f}), x_{j}}^{(j)}$. Mixtures of multinomials are quite effective for modeling multivariate categorical data distributions (Dunson and Xing, 2009). As desired, the model can capture dependencies between $\mathbf{X}^{(R)}$ and $\mathcal{F}$, as the weights and mixture component parameters depend on $\mathcal{F}$.

The $f\left(\mathbf{W}^{(R)}, \mathbf{Z}^{(R)} \mid \mathbf{X}^{(R)}, \mathcal{F}\right)$ is a mixture of multivariate normal linear regressions, with means that are functions of $\left(\mathbf{X}^{(R)}, \mathcal{F}\right)$ and weights that are functions of $\mathcal{F}$. We have

$$
f\left(\mathbf{W}^{(R)}, \mathbf{Z}^{(R)} \mid \mathbf{X}^{(R)}=\boldsymbol{x}, \mathcal{F}=\boldsymbol{f}\right)=\sum_{h=1}^{N(\boldsymbol{f})} p_{h}(\boldsymbol{f}) \mathrm{N}\left(\mathbf{W}^{(R)}, \mathbf{Z}^{(R)} ; \boldsymbol{D}(\boldsymbol{x}, \boldsymbol{f}) \boldsymbol{\beta}_{\pi_{h}(\boldsymbol{f})}, \boldsymbol{\Sigma}_{\pi_{h}(\boldsymbol{f})}\right) .
$$

From (6) we can integrate out $\mathbf{Z}^{(R)}$ and obtain the probability that $\mathbf{Y}^{(R)}$ takes on a particular combination of ordinal levels $\left(y_{1}, \ldots, y_{p_{o}}\right)$ conditional on $\mathcal{F}$ and $\mathbf{X}^{(R)}$. This gives $\operatorname{Pr}\left(\mathbf{Y}^{(R)}=\right.$ $\left.\left(y_{1}, \ldots, y_{p_{o}}\right) \mid \mathbf{X}^{(R)}=\boldsymbol{x}, \mathcal{F}=\boldsymbol{f}\right)=$

$$
\sum_{l=1}^{N(\boldsymbol{f})} p_{l}(\boldsymbol{f}) \int_{\gamma_{p_{o}, y_{p_{o}}-1}}^{\gamma_{p_{o}, y_{p_{o}}}} \cdots \int_{\gamma_{1, y_{1}-1}}^{\gamma_{1, y_{1}}} \mathrm{~N}\left(\boldsymbol{W}^{(R)} \mid\left(\boldsymbol{D}(\boldsymbol{x}, \boldsymbol{f}) \boldsymbol{\beta}_{\pi_{l}(\boldsymbol{f})}\right)^{(W)}, \boldsymbol{\Sigma}_{\pi_{l}(\boldsymbol{f})}^{(W)}\right) d \boldsymbol{W}^{(R)},
$$

where the superscript $(W)$ indicates the portion of $\boldsymbol{D}(\boldsymbol{x}, \boldsymbol{f}) \boldsymbol{\beta}_{\pi_{l}(\boldsymbol{f})}$ and $\boldsymbol{\Sigma}_{\pi_{l}(\boldsymbol{f})}$ that corresponds to $\boldsymbol{W}^{(R)}$.

The weights in (6) and (7) reveal additional flexibility that results from using the LDP. Since the weights are dependent on $\mathcal{F}$, CMM-Mix is able to capture relationships between $\left(\mathbf{Y}^{(R)}, \mathbf{Z}^{(R)}\right)$ and $\mathcal{F}$ beyond those implied by $\boldsymbol{D}\left(\mathbf{X}^{(R)}, \mathcal{F}\right)$. This additional flexibility does not extend to relationships between $\left(\mathbf{Y}^{(R)}, \mathbf{Z}^{(R)}\right)$ and $\mathbf{X}^{(R)}$.

\subsection{Specifying the distance function $d(\cdot, \cdot)$ and the value of $d^{*}$}

We base $d(\cdot, \cdot)$ on Gower's generalized coefficient of dissimilarity (Gower, 1971), which is a standard dissimilarity measure for mixed data (Kaufman and Rousseeuw, 1990, Maimon and Rokach, 2010). The distance between two $q \times 1$ vectors $\boldsymbol{f}$ and $\boldsymbol{f}^{\prime}$, or $d\left(\boldsymbol{f}, \boldsymbol{f}^{\prime}\right)$, is a weighted 
sum of the element-wise distances, $\sum_{j=1}^{q} w_{j} d_{j}\left(f_{j}, f_{j}^{\prime}\right)$. Each $d_{j}$ takes values between 0 and 1 , and $\sum_{j=1}^{q} w_{j}=1$ with each $w_{j} \geq 0$. For ordinal values, $d_{j}\left(f_{j}, f_{j}^{\prime}\right)=\left|f_{j}-f_{j}^{\prime}\right| /\left(k_{j}^{(R)}-1\right)$. For continuous values, $d\left(f_{j}, f_{j}^{\prime}\right)=\left|f_{j}-f_{j}^{\prime}\right| / \operatorname{range}\left(f_{j}\right)$. For nominal values, we use the Hamming distance, $d_{j}\left(f_{j}, f_{j}^{\prime}\right)=1$ when $f_{j} \neq f_{j}^{\prime}$, and $d_{j}\left(f_{j}, f_{j}^{\prime}\right)=0$ when $f_{j}=f_{j}^{\prime}$. Hence, values of $d\left(\boldsymbol{f}, \boldsymbol{f}^{\prime}\right)$ near zero indicate similar $\left(\boldsymbol{f}, \boldsymbol{f}^{\prime}\right)$, and values near one indicate otherwise.

When $\mathcal{F}$ comprises survey design variables or when $q$ is modest, we set $w_{j}=1 / q$ for $j=1, \ldots, q$. This default assignment gives each variable equal weight in determining similarities. However, one can improve computational efficiency and possibly inferential accuracy by using only a subset of variables in $d\left(\boldsymbol{f}, \boldsymbol{f}^{\prime}\right)$; that is, by setting $w_{j}=0$ for some $j$. In particular, it may be beneficial to set $w_{j}=0$ for variables in $\mathcal{F}$ that do not contribute meaningfully to predicting $\mathbf{X}^{(R)}$, since the primary function of the LDP is to connect $\mathbf{X}^{(R)}$ and $\mathcal{F}$. Additionally, one can set $w_{j}=0$ for one or more $\mathcal{F}$ variables that are highly predictive of one another.

To perform feature selection (Guyon and Elisseeff, 2003) and determine which $w_{j}=0$, we rely on values of mutual information, which can be used to describe dependencies between random variables of any type without assumptions about the nature of their underlying relationships (Battiti, 1994). For generic discrete random variables $A$ and $B$, their mutual information is $I(A, B)=\sum_{a} \sum_{b} p(a, b) \log \{p(a, b) /(p(a) p(b))\}$; values near zero indicate only weak dependence between $A$ and $B$. In our context, the value of $I\left(\mathcal{F}_{l}, X_{j}^{(R)}\right)$ for any $(l, j)$ is, intuitively speaking, the amount of uncertainty in $X_{j}^{(R)}$ that is explained by $\mathcal{F}_{l}$. We estimate these quantities to derive a single measure of the simultaneous explanatory power of $\mathcal{F}_{l}$ on $\mathbf{X}^{(R)}$, namely $I_{\mathcal{F}_{l}, x}^{\max }=\max \left\{I\left(\mathcal{F}_{l}, X_{1}^{(R)}\right), \ldots, I\left(\mathcal{F}_{l}, X_{p_{n}}^{(R)}\right)\right\}$. Based on the estimated values of $I_{\mathcal{F}_{l}, x}^{\max }$, we use a forward selection procedure to select the set of variables in $\mathcal{F}_{l}$ having $w_{l}>0$, i.e., those deemed to have explanatory power beyond a threshold, and let the complementary set have $w_{l}=0$. Details of the algorithm are in Appendix A.2.

We still include variables with $w_{j}=0$ in the regression for $\left(\mathbf{W}^{(R)}, \mathbf{Z}^{(R)}\right)$. If not, we would be forcing these variables to be conditionally independent of the random variables. Even when mutual information values suggest weak dependence with $\mathbf{X}^{(R)}, \mathcal{F}_{j}$ still may be 
predictive of $\left(\mathbf{W}^{(R)}, \mathbf{Z}^{(R)}\right)$. We note that including irrelevant variables in $\boldsymbol{D}\left(\mathbf{X}^{(R)}, \mathcal{F}\right)$ is not problematic computationally (when $q$ is not huge), so that we prefer not to force conditional independence a priori.

We also must specify the neighborhood size $d^{*}$. As a benchmark, consider what happens when $d^{*} \rightarrow 1^{-}$. In this case, all $\boldsymbol{\Gamma}_{h}$ are in the neighborhood of any value of $\mathcal{F}$, so that $H_{i}$ in (3) is simply drawn from $\{1, \ldots, N\}$ as in the usual Bayesian mixture model. Hence, to facilitate sharing of components by records with similar values of $\mathcal{F}$, we seek a $d^{*}$ away from one. One approach is to determine a $d^{*}$ so that each observation belongs to the same neighborhood as $r \%$ of the observations on average, for instance $r=20$ (Chung and Dunson, 2011). Alternatively, we can base $d^{*}$ on interpretations of the distance function. For instance, with all nominal $\mathcal{F}$, setting $d^{*}=0.5$ implies that observations must exactly match on at least $50 \%$ of values to be in the same neighborhood and share mixture components. In our data applications, the results were insensitive to different reasonable values of $d^{*}$.

\subsection{Posterior inference and missing data considerations}

For posterior inference, we use a Gibbs sampler based on the finite stick-breaking representation of the DP (Ishwaran and James, 2001). With missing values, the sampler proceeds via data augmentation, i.e., given a draw of the parameters, we draw new values of the missing data. We present the posterior full conditionals in Appendix A.3. After MCMC convergence, analysts can use the completed datasets for multiple imputation inferences $(\mathrm{Rubin}, 1987 ; \mathrm{Hu}$ et al. 2013), or directly make posterior inferences from relevant functions of the parameters.

Missing data can cause numerical problems in the algorithm, namely that elements of $\boldsymbol{\beta}_{h}$, $\boldsymbol{\Sigma}_{h}$, and $\boldsymbol{\tau}$ can diverge towards very large values. In particular, problems occur when the mixture model samples clusters with all observed values of some ordinal variable equal to the

first or last category, e.g., $Y_{i j}^{(R)}=1$ for all observed $Y_{i j}^{(R)}$ in some cluster. This creates a perfect prediction problem and ensuing estimation difficulties. In our example, the mixture model will strongly favor imputing category 1 for the missing values of $Y_{i j}^{(R)}$ in the cluster. Hence, it will favor making the corresponding latent $W_{i j}$ very negative, which happens when elements 
of $\boldsymbol{\beta}_{h}$ are large in magnitude. This in turn can cause some elements of $\boldsymbol{\tau}$ to become extremely large. Related problems arise with other types of perfect predictions within clusters. We note that these problems can arise in any mixture model (not just CMM-Mix) that uses probit specifications for ordinal variables.

We use a quick and dirty fix that prevents elements of $\boldsymbol{\beta}_{h}$ from getting too large. Given the standardization of all continuous variables and choice of cut-offs for the latent continuous random variables, practically we need not allow elements of $\left|\boldsymbol{\beta}_{h}\right|$ to exceed 4 or 5 , as this still

allows the average value of each $\mathbf{Y}_{j}^{(R)}$ to be as small as 1 or as large as $k_{j}^{(R)}$. We therefore restrict $\tau_{j}^{2} \leq 6$ via a truncated inverse-gamma prior in place of an inverse-gamma prior. This keeps the parameters from diverging to large values without being overly restrictive, since the draws for $\tau_{j}^{2}$ are centered far to the left of the truncation value in all of our model implementations. We include plots of posterior samples for $\tau_{j}^{2}$ in the supplementary material.

\section{Evaluating CMM-Mix using a data fusion scenario}

In data fusion, analysts seek to combine information from two or more databases containing information on disjoint sets of individuals. For example, a set of demographic variables $A$ is available in two databases $D_{1}$ and $D_{2}$, a set of variables $B_{1}$ is available only in $D_{1}$, and a set of variables $B_{2}$ is available only in $D_{2}$. The analyst seeks to use $D_{1}$ and $D_{2}$ to learn about the joint distribution of all variables. Without simultaneous observations of $\left\{A, B_{1}, B_{2}\right\}$, the analyst is forced to make identifying assumptions about the conditional associations between $B_{1}$ and $B_{2}$ given $A$. The simplest and most common assumption is that $B_{1}$ and $B_{2}$ are conditionally independent given $A$, which may be reasonable when $A$ is rich.

Kamakura and Wedel (1997) proposed that data fusion be implemented by fitting mixture models to the concatenation of $\left(D_{1}, D_{2}\right)$, so as to capture nonstandard distributions and complex associations among $\left(A, B_{1}\right)$ and $\left(A, B_{2}\right)$ automatically. However, Gilula et al. (2006) questioned whether or not mixture models for data fusion truly encode conditional independence between $B_{1}$ and $B_{2}$. Indeed, Fosdick et al. (2016) find in simulations that a mixture model generated stronger estimated associations between $B_{1}$ and $B_{2}$ than implied 
by conditional independence.

These results motivate the simulation studies of this section. Specifically, we investigate whether or not CMM-Mix more faithfully respects conditional independence than a fully joint mixture model in data fusion contexts. Viewed more generally, we compare how well CMM-Mix and a fully joint mixture model estimate true joint distributions in the presence of missing data. We also performed a separate empirical study with arbitrary itemwise missing data patterns; results and conclusions, available in the supplementary material, indicate that CMM-Mix provides accurate inferences.

\subsection{Constructing the data fusion scenario}

We base the simulations on data from a survey of $n=3567$ individuals collected by the book publisher HarperCollins. We treat eleven variables from the survey as $A$ variables, including six ordinal variables (age, passion for books, reading hours, income, passion for Internet, and opinion on romance in books) and five nominal variables (work status, importance that books that challenge the reader, laptop ownership, eBook reader ownership, use of audiobooks). To construct a data fusion scenario where conditional independence is known to hold, we generate a continuous variable $Z$, an ordinal variable $Y$, and a nominal variable $X$ such that $f(Z, Y, X \mid A)=f(Z \mid A) f(Y \mid A) f(X \mid A)$. We use normal, probit, and multinomial logistic regressions to generate $Z, Y$, and $X$, respectively, using various main effects and interactions involving only $A$ in the predictor functions. The exact specifications are presented in the supplementary material. After binding these simulated variables to $A$, we blank $(X, Z)$ for the first 1189 rows, $(X, Y)$ for the second 1189 rows, and $(Y, Z)$ for the final 1189 rows. This simulates a data fusion scenario with three databases. This process is repeated 40 times to create 40 unique sets of databases to be fused, which results in Monte Carlo standard errors that are adequately small for our purposes. 


\subsection{Results}

We consider the eleven $A$ variables as $\mathcal{F}$, and $(Z, Y, X)$ as $\mathcal{R}$. In one randomly chosen

set of simulated $\mathcal{R}$, the (normalized) values of $I_{\mathcal{F}_{l}, x}^{\max }$ to two decimal places in descending order are $(.25, .23, .07, .07, .06, .06, .02, .02, .01, .01, .00)$; other replications yield similar values. Given the number of weak associations, we investigate two possibilities for assigning nonzero weights in $d(\cdot, \cdot)$. First, we allow only the top two scoring variables - reading hours (ordinal with 5 categories) and laptop ownership (binary) - to have $w_{j}>0$; this includes only moderately strong predictors. Second, we allow the top six scoring variables - ordinal variables age, passion for books, and income, and binary variable desire for challenge in reading - to have $w_{j}>0$; this includes variables that are weakly predictive. The maximum normalized mutual information between any $\left(\mathcal{F}_{l}, \mathcal{F}_{j}\right)$ pair is 0.27 , corresponding to passion for books and reading hours per week, suggesting no serious redundancies among $\mathcal{F}$.

For each feature selection, we consider three sets of possible $d^{*}$ values. For the two feature model, we consider $d^{*} \in(0.5,0.25,0.125)$. Here, 0.5 is the maximum distance between two individuals' vectors of reading hours and laptop ownership when only one of the two variables matches. The distance is 0.125 when the laptop ownership matches and reading hours is separated by just one category. This is the most stringent criterion besides requiring that both variables must match exactly, which would set $d^{*}=0$. The 0.25 is an intermediate value. These $d^{*}$ values imply respectively that each observation is in the same neighborhood as $66 \%, 48 \%$ and $34 \%$ of the other observations on average. For the six variable feature selection, we consider $d^{*} \in(0.375,0.30,0.25)$. These $d^{*}$ values imply that each observation is in the same neighborhood as $52 \%, 34 \%$, and $23 \%$ of the other observations on average.

We fit the six versions of CMM-Mix to each concatenated dataset on $\{Y, Z, X, A\}$ containing missing values. We refer to the two feature models as $\mathrm{C}-2 \mathrm{~S}, \mathrm{C}-2 \mathrm{M}$, and $\mathrm{C}-2 \mathrm{~L}$, where the $\mathrm{S}, \mathrm{M}$, and $\mathrm{L}$ indicate the smallest to largest values of $d^{*}$, respectively. We refer to the six feature models as C-6S, C-6M, and C-6L using analogous nomenclature. We also fit a fully joint mixture model that puts all fourteen variables in $\mathcal{R}$. For each method, we create 10 completed data sets for use in multiple imputation inferences. 


\begin{tabular}{l|c|c|c|c|c|c|c} 
& Joint & C-2S & C-2M & C-2L & C-6S & C-6M & C-6L \\
\hline \% of CIs Covering & .655 & .905 & .813 & .792 & .854 & .839 & .791 \\
Mean Abs. Error & .0128 & .0074 & .0066 & .0081 & .0066 & .0074 & .0087 \\
$25 \%$ ile Abs. Error & .0025 & .0016 & .0012 & .0011 & .0013 & .0013 & .0013 \\
$75 \%$ ile Abs. Error & .0161 & .0097 & .0086 & .0090 & .0089 & .0091 & .0100
\end{tabular}

Table 1: Summary of inference for cells corresponding to bivariate discrete distributions with missing data. Entries include the proportions of $95 \%$ multiple imputation confidence intervals (CIs) that contain the truth, and the mean, 25th percentile, and 75th percentile of absolute errors, averaged over all 40 datasets.

We begin with inferences for all 285 cell probabilities associated with the bivariate distributions of $\left(X, A_{j}\right)$ and $\left(Y, A_{j}\right)$ for all $j$. For each of the 40 simulated datasets, we calculate the proportion of multiple imputation $95 \%$ confidence intervals that contain their corresponding ground truths. We also calculate summaries of the absolute errors of the point estimates, including the mean, 25th percentile, and 75th percentile. As evident in Table 1, the joint model results in the lowest coverage rates and largest absolute errors. Differences are unlikely due to Monte Carlo error, as the standard errors of reported quantities across the 40 sets of results are relatively small; for example, the estimated standard errors of all coverage rates for the conditional models are less than .006, and the standard error of the coverage rates for the joint model is .016.

The models with small $d^{*}$ values result in the largest coverage rates. The absolute errors are generally smallest under the small and medium $d^{*}$ values, but all CMM-Mix models tend to be more accurate than the joint model. The models with small $d^{*}$ outperform those with large $d^{*}$, highlighting the benefits of dependent cluster assignments.

Turning to $Z$, inferences for its mean, 25th percentile, and 75th percentile are more accurate under the CMM-Mix models than the joint model. Almost all 95\% CIs from the conditional models contain the true mean zero, with all average point estimates less than .027 in absolute value. By comparison, the joint model produces an average point estimate of 


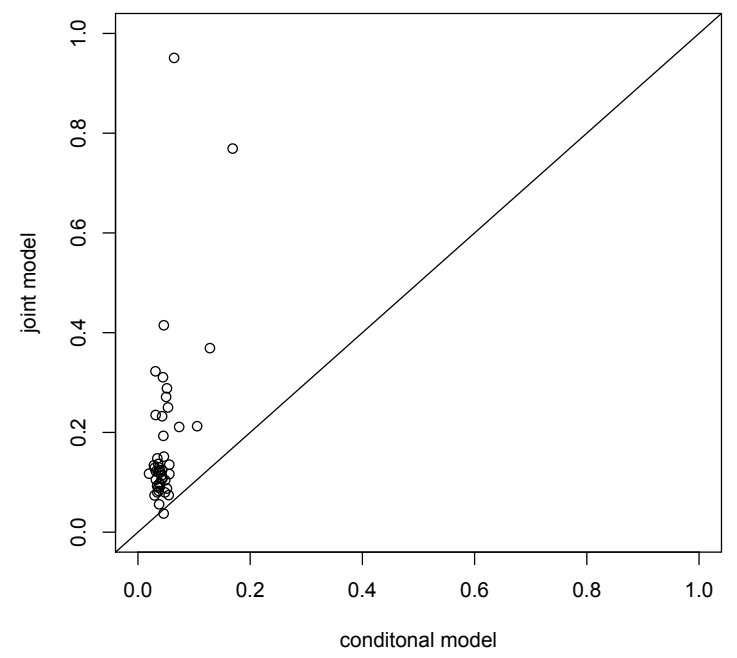

Figure 1: Average absolute errors of 46 multiple imputation point estimates for the mean of $Z$ conditional on each $A$ variable from the joint model and C-2S.

.093 , and more than half of the $95 \%$ CIs do not contain zero. We also estimate $\mathrm{E}\left(Z \mid A_{j}\right)$ for each value of $A_{j}$ for all $j$. Once again, the joint model results in noticeably larger errors than the CMM-Mix models. The average absolute errors of the 46 point estimates from the joint model versus C-2S are shown in Figure 1. Figures for other models are in the supplementary material.

We next investigate which mixture models best estimate the generation model for $(X, Y, Z) \mid$ $A$; in other words, which models are most faithful to the conditional independence assumption for data fusion. Here we focus on one randomly selected simulation run for simplicity in presentation of results. For each of the 10 completed data sets, we fit a regression model of $Z$ conditional on $X$ and $Y$, as well as all variables in $A$ that actually generated the data. We use multiple imputation inference to create point estimates and $95 \%$ confidence intervals for all regression coefficients. We also fit the model to the fully observed data without any missing values, referred to as the pre-missing data, and obtain the true empirical $95 \%$ confidence intervals for regression coefficients.

With the full joint model, the $95 \%$ confidence intervals for three of the four regression 
coefficients for $X$ and $Y$ do not contain the true value of zero, whereas with all versions of CMM-Mix and the pre-missing data all intervals contain zero. All models result in confidence intervals that exclude zero for ten of the eleven non-zero coefficients involving $A$. The average absolute error of the regression coefficient estimates from the joint model is .252, whereas the averages are less than .08 for all CMM-Mix models. Eleven of the sixteen regression coefficient confidence intervals from the joint model do not even overlap with their corresponding confidence intervals based on the pre-missing data; in contrast, all sixteen confidence intervals from CMM-Mix fully contain their corresponding confidence intervals based on the pre-missing data. We find similar results when fitting regression models that treat $Y$ or $X$ as the response. We also validate that the joint model is less consistent with conditional independence assumptions than the CMM-Mix models using the strategy of Kunihama and Dunson (2016) based on conditional mutual information. Details of all additional results are in the supplementary material.

Finally, we investigate the performance of statistical matching (van der Putten et al. 2002 Wicken and Elms, 2009), a common technique used for data fusion. Here, we create fused data sets via exact matching on the $11 A$ variables using the StatMatch package in $\mathrm{R}$ (D'Orazio, 2015). For each record $i$ with missing $Y_{i}$, this method identifies all observations $\left\{i^{\prime} \neq i: Y_{i^{\prime}}\right.$ is observed $\}$ with the smallest Hamming distance from $A_{i}$, and samples one of their $Y$ values as an imputation for the missing $Y_{i}$. It operates similarly for observations missing $X$ or $Z$. Using this method, on average, only $65 \%$ of confidence intervals associated with bivariate probabilities contain the true values. Thus, the statistical matching approach is not as effective as the conditional mixture model approaches.

In summary, the results suggest that the imputations for the variables with missing data from the joint mixture model are not consistent with conditional independence, whereas those from the CMM-Mix models are. Additionally, the CMM-Mix models estimate the relationships in the data-generating model as or more reliably than the joint model. There is evidence of potential for inferential gains by choosing smaller $d^{*}$ and a more parsimonious set of features driving the cluster assignments. 


\section{Conditional Inference from a Quota Sample}

We now analyze a quota sample conducted by HarperCollins in 2013 to learn about U. S. consumers' reading behaviors and interests. The sample comprises $n=3631$ individuals, sampled to achieve fixed quotas in various age, gender, ethnicity, and region (location) groups. We seek inferences for the relationships among income, passion for books, passion for the Internet, reading hours, laptop ownership, eBook ownership, and desire to be challenged in reading. Most variables are complete; only income and reading hours have isolated missingness, which we assume to be at random. Income, passion for books and the Internet, and

reading hours are ordinal and comprise $\mathbf{Y}^{(R)}$; eBook ownership and laptop ownership are nominal and comprise $\mathbf{X}^{(R)}$; and, the measure of desire for challenging books is numerical and comprises $Z^{(R)}$. We condition on all the design variables when fitting CMM-Mix, letting $\mathcal{F}$ include age, gender, ethnicity and region with all $w_{j}>0$.

We fit CMM-Mix with $d^{*}=0.25$, which implies that each observation is in the same neighborhood as $14 \%$ of the other observations on average, and observations must match on least of 3 of the 4 design variables to be in the same cluster. Posterior predictive model checks do not suggest evidence of lack of model fit; results are in the supplementary material. We focus on full posterior inference rather than multiple imputation inference, as some subgroups (i.e., combinations of age, gender, ethnicity, region) have few or no individuals in sample. We make conditional inferences rather than marginal inferences, as we do not have population distributions of the quota variables.

We focus inferences on individuals aged 25-34, one of the most sought after demographics in marketing (Madrigal, 2012; Cocotas, 2012). In particular, we contrast the characteristics of individuals in this group who own eBook readers and those who do not own eBook readers. CMM-Mix enables inferences about many other demographic groups; we report only on one here to present a concise and coherent analysis.

Figure 2 displays the distribution of income for eBook reader owners and non-owners for white females aged 25-34 living in the South. Owners of eBook readers tend to have higher incomes than non-owners, with the most striking difference in the percentage of individuals 

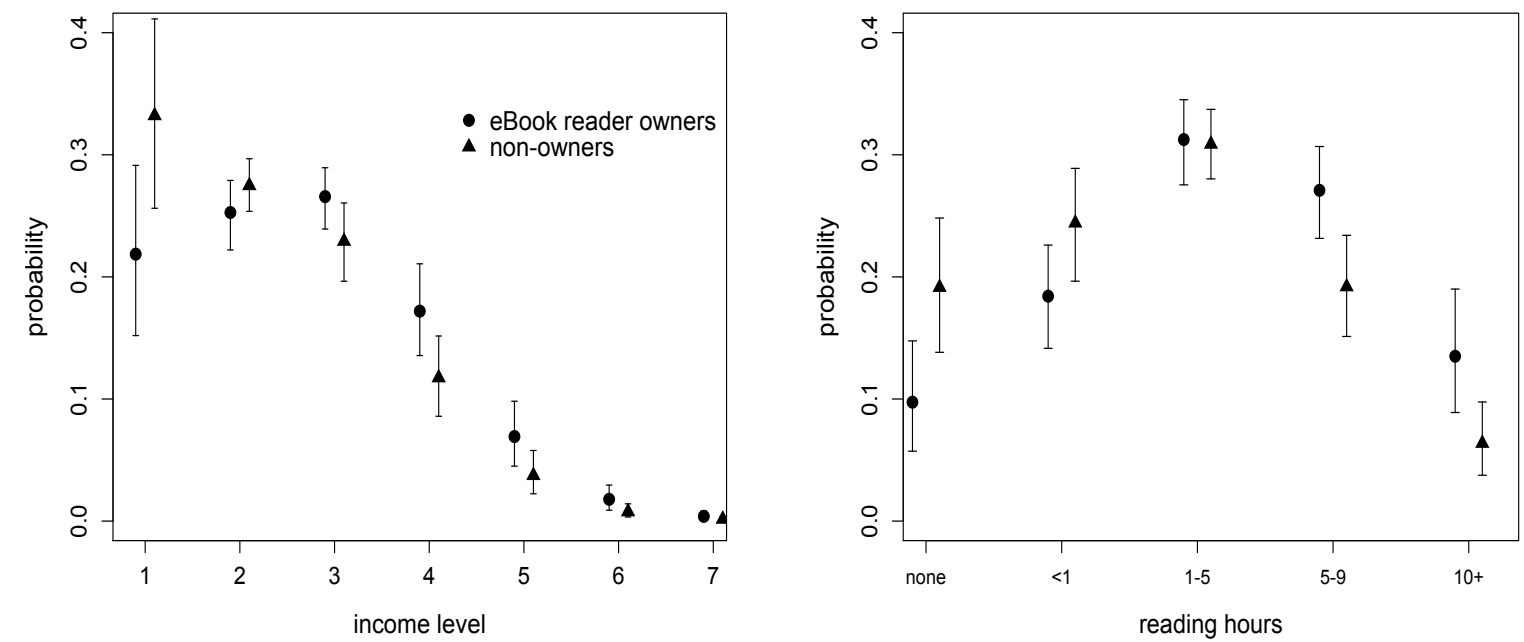

Figure 2: Left: Posterior mean and 90\% credible intervals for the probability assigned to each income level for eBook owners (circle symbols) and non-owners (triangle symbols). Income levels 1 through 7 correspond to < 25K, 25-44K, 45-74K, $75-99 \mathrm{~K}, 100-149 \mathrm{~K}, 150-199 \mathrm{~K}$, $\geq 200 \mathrm{~K}$. Right: Posterior mean and 90\% credible intervals for the probability assigned to each level of weekly reading hours for owners versus non-owners. Both displays refer to white females aged 25-34 living in the South.

making less than $\$ 25000$ per year. We obtain these posterior inferences using expression (7), integrating out the nominal random variable laptop, $X_{2}^{(R)}$, using the estimates for $\operatorname{Pr}\left(X_{2}^{(R)}=\right.$ $x)=\sum_{l=1}^{N(\mathcal{F})} p_{l}(\mathcal{F}) \psi_{\pi_{l}(\mathcal{F}), x}^{(2)}$. Figure 2 also displays the posterior distribution of weekly reading hours for owners and non-owners. Overall, owners of eBook readers tend to spend more hours reading than non-owners, and are noticeably less likely to spend zero hours reading.

These results are in accord with prior research suggesting that higher income individuals are more likely to be frequent readers (Jansen, 2010; Rainie et al., 2012). Research also suggests that higher income individuals use the Internet more frequently. Building on these findings, we next contrast eBook owners' and non-owners' views on the importance of books and of the Internet, drilling down by race to provide additional information. Figure 3 displays the probability that individuals aged 25-34 from the South regard books as very important 
in their lives, and the probability that individuals aged 25-34 regard the Internet as very important in their lives, as functions of gender, race, and eBook reader ownership. The posterior inferences suggest that, regardless of income level, white females are most likely to view books as important, and white males are the least likely to view books as important. As might be expected, those who own eBook readers are more likely to view books as very important than those who do not own eBook readers. The posterior inferences also suggest that, regardless of income level, black males are most likely to view the Internet as important, and white females are least likely to view the Internet as important. The results suggest that the importance of the Internet tends to increase as a function of income for both eBook reader owners and non-owners, except for white females. The model suggests little to no interactions between gender and eBook ownership, and race and eBook ownership. Exploratory analysis and posterior predictive checks are consistent with these findings, indicating this is not some artifact of the model specification.

Finally, we examine whether or not eBook reader owners prefer books that challenge them to think more than those who do not own eBook readers. This involves posterior distributions of the continuous variable "challenge", conditional on eBook reader ownership for each combination of age, gender, ethnicity, and region. Larger values of "challenge" indicate increasing appreciation for books that challenge the reader to think. The distribution $f\left(Z^{(R)} \mid \mathcal{F}, \mathbf{X}^{(R)}\right)=\sum_{l=1}^{N(\mathcal{F})} p_{l}(\mathcal{F}) \mathrm{N}\left(Z^{(R)} \mid\left(\boldsymbol{D}\left(\mathbf{X}^{(R)}, \mathcal{F}\right) \boldsymbol{\beta}_{\pi_{l}(\mathcal{F})}\right)^{(Z)}, \boldsymbol{\Sigma}_{\pi_{l}(\mathcal{F})}^{(Z)}\right)$, where the superscript $(Z)$ indicates the portion of $\boldsymbol{D}\left(\mathbf{X}^{(R)}, \mathcal{F}\right) \boldsymbol{\beta}_{\pi_{l}(\mathcal{F})}$ and $\boldsymbol{\Sigma}_{\pi_{l}(\mathcal{F})}$ that corresponds to $Z^{(R)}$. To estimate $f\left(Z^{(R)} \mid X_{1}^{(R)}, \mathcal{F}\right)$, where $X_{1}^{(R)}$ refers to the random variable "eBook ownership", we integrate out $X_{2}^{(R)}$ using the estimates for $\operatorname{Pr}\left(X_{2}^{(R)}=x\right)=\sum_{l=1}^{N(\mathcal{F})} p_{l}(\mathcal{F}) \psi_{\pi_{l}(\mathcal{F}), x}^{(2)}$.

Figure 4 displays mean posterior distributions of the challenge variable for four combinations of age, gender, ethnicity, and region. The shapes of the distributions are varied, with some having skewness or bimodality and others being standard unimodal distributions. For almost all combinations of age, gender, ethnicity, and region, the distribution of the challenge variable is centered on larger values for eBook reader owners than for non-owners. This suggests that those who own eBook readers tend to prefer books that are challenging 

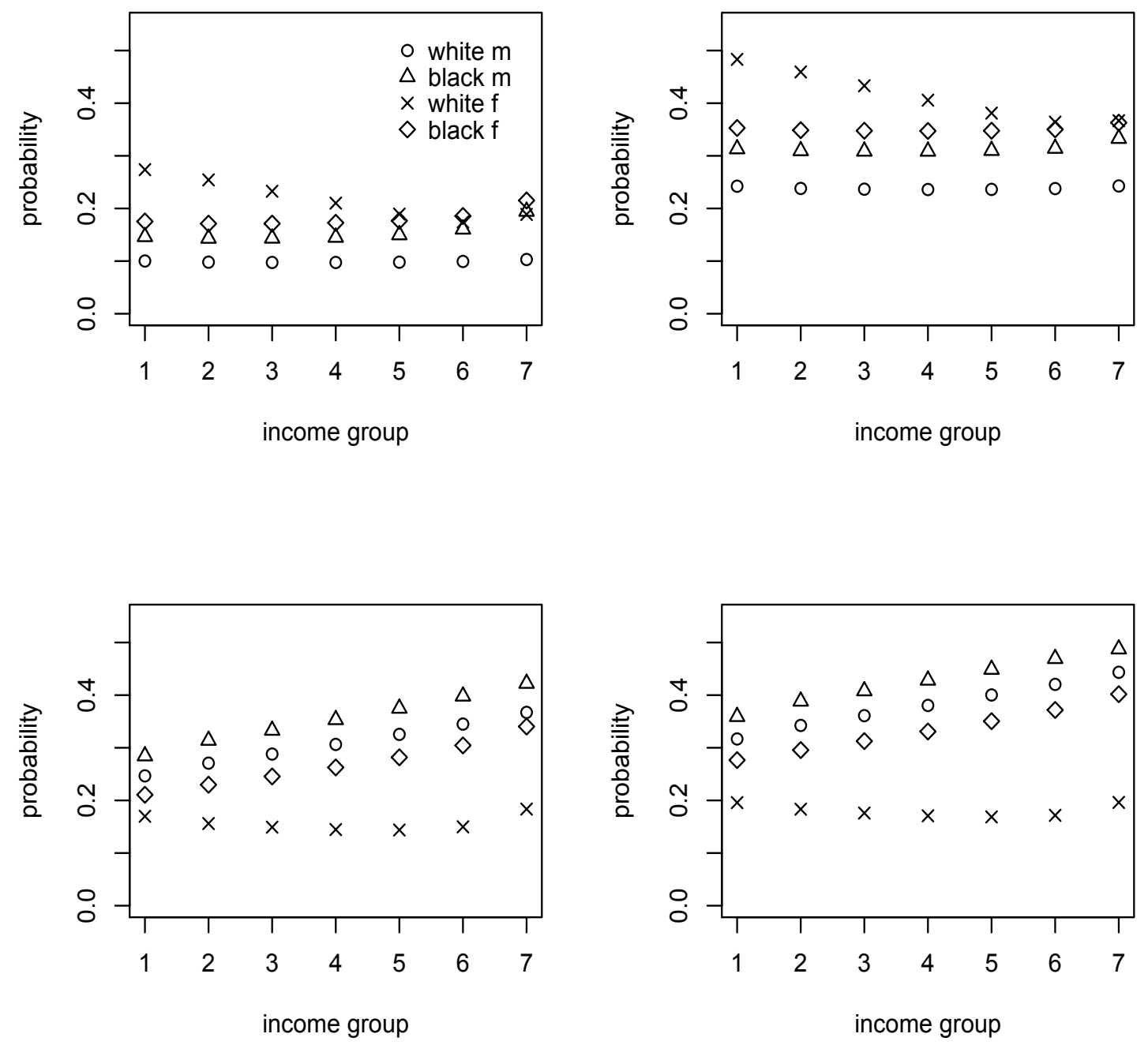

Figure 3: Posterior mean estimates for the probability that one views books as very important (top row) and for the probability that one views the Internet as very important (bottom row) as a function of income, gender, and race, for eBook non-owners (left column) and owners (right column). Income levels 1 through 7 correspond to $<25 \mathrm{~K}, 25-44 \mathrm{~K}, 45-74 \mathrm{~K}, 75-99 \mathrm{~K}$, $100-149 \mathrm{~K}, 150-199 \mathrm{~K}, \geq 200 \mathrm{~K}$. 

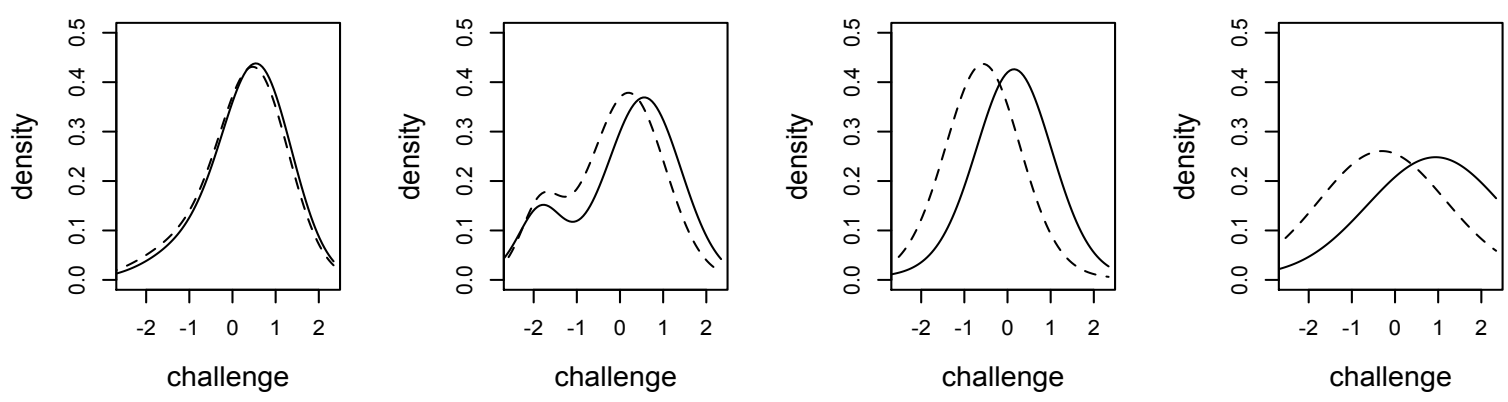

Figure 4: Mean posterior distribution of the "challenge" variable given that one owns an eBook (solid) or does not own an eBook (dashed). Figures refer to four combinations of age, gender, ethnicity, and region. Left to right: 25-34, female, white, South; 25-34, male, white, Northeast; 35-44, female, Hispanic, West; 45-54, male, other, West.

as compared to those who do not own eBook readers.

All of these inferences are conditional on fixed quota variables. For unconditional inferences, for instance national estimates, analysts can integrate out $\mathcal{F}$ over its distribution in the population. In our context, this can be accomplished by estimating the quantity of interest for each combination of age, gender, ethnicity, and region, and averaging over these estimates according to each group's population share. For inferences conditional on only age and region, for example, we marginalize over the distribution of ethnicity and gender in the population of U. S. individuals aged 18 and older.

\section{Discussion}

We conclude with a discussion of future research directions, beginning with the feature selection algorithm for the LDP. The simulations suggest that when the number of variables in $\mathcal{F}$ is large, one can improve accuracy by selecting only a subset of the variables to include in the distance calculation. We used equal weights for features selected to be in the distance function. It may be beneficial to allow the non-zero weights to differ, for example to be proportional to the values of mutual information with the $\mathbf{X}^{(R)}$ variables. 
The CMM-Mix models also may point an alternative path for handling survey weights in Bayesian mixture models inference, which is an active area of research (e.g., Kunihama et al., 2016; Si et al., 2015, Savitsky et al., 2016). Since the survey weights are fixed and fully observed, one could include them as an $\mathcal{F}$ variable. This would make the conditional distribution for the random variables a function of the survey weights, as is done in the Gaussian process model of Si et al. (2015). Here, observations with similar survey weights would be encouraged to share mixture components.

Finally, our work with CMM-Mix reveals problems when using mixture models to impute missing ordinal values that can be explored further. Specifically, the mixture models sometimes favor clusters that are homogeneous in observed values, which encourages imputations also to be at the observed values. This can be undesirable, as it may result in underestimation of imputation uncertainty, particularly when cluster assignments are relatively stable. It may be beneficial somehow to add uncertainty into homogeneous clusters. Related ideas are used by Paiva and Reiter (2015), who adjust the weights associated with mixture components in order to generate imputations that follow a nonignorable missing data mechanism.

\section{References}

Banerjee, A., Murray, J., and Dunson, D. (2013), "Bayesian learning of joint distributions of objects," in Proceedings of the 16th International Conference on Artificial Intelligence and Statistics.

Battiti, R. (1994), "Using mutual information for selecting features in supervised neural net learning," IEEE Transactions on Neural Networks, 5, 537-550.

Chung, Y. and Dunson, D. (2011), "The local Dirichlet process," Annals of the Institute of Statistical Mathematics, 63, 59-80.

Cocotas, A. (2012), "Young women are the most valuable mobile ad demographic," www. businessinsider.com/young-women-are-most-valuable-mobile-ad-demographic2012-2. 
De Iorio, M., Müller, P., Rosner, G., and MacEachern, S. (2004), "An ANOVA model for dependent random measures," Journal of the American Statistical Association, 99, 205215.

Ding, C. and Peng, H. (2003), "Minimum redundancy feature selection from microarray gene expression data," in Proceedings of the 2003 IEEE, pp. 523-528.

D’Orazio, M. (2015), StatMatch: Statistical Matching, R package version 1.2.3.

D'Orazio, M., Di Zio, M., and Scanu, M. (2006), Statistical Matching: Theory and Practice, New York: Wiley.

Dunson, D. and Bhattacharya, A. (2010), "Nonparametric Bayes regression and classication through mixtures of product kernels," Bayesian Statistics, 9, 145-164.

Dunson, D. and Park, J. (2008), "Kernel stick-breaking processes," Biometrika, 95, 307-323.

Dunson, D. and Xing, C. (2009), "Nonparametric Bayes modeling of multivariate categorical data," Journal of the American Statistical Association, 104, 1042-1051.

Eztévez, P., Tesmer, M., and Zurada, J. (2009), "Normalized mutual information feature selection," IEEE Transactions on Neural Networks, 20, 189-201.

Fosdick, B., DeYoreo, M., and Reiter, J. (2016), "Categorical data fusion using auxiliary information," Annals of Applied Statistics, To appear.

Gilula, Z., McCulloch, R., and Rossi, P. (2006), "A direct approach to data fusion," Journal of Marketing Research, 43, 73-83.

Gower, J. C. (1971), "A general coefficient of similarity and some of its properties," Biometrics, $27,857-871$.

Griffin, J. and Steel, M. (2006), "Order-based dependent Dirichlet processes," Journal of the American Statistical Association, 101, 179-194. 
Guyon, I. and Elisseeff, A. (2003), "An introduction to variable and feature selection," Journal of Machine Learning Research, 3, 1157-1182.

Hannah, L., Blei, D., and Powell, W. (2011), "Dirichlet process mixtures of generalized linear models," Journal of Machine Learning Research, 1, 1-33.

Hu, J., Mitra, R., and Reiter, J. (2013), "Are independent draws necessary for multiple imputation?" The American Statistician, 67, 143-149.

Ishwaran, H. and James, L. (2001), "Gibbs sampling methods for stick-breaking priors," Journal of the American Statistical Association, 96, 161-173.

Jansen, J. (2010), "Use of the internet in higher-income households," http://www . pewinternet .org/2010/11/24/use-of-the-internet-in-higher-incomehouseholds.

Kamakura, W. and Wedel, M. (1997), "Statistical data fusion for cross tabulation," Journal of Marketing Research, 34, 485-498.

Kaufman, L. and Rousseeuw, P. (1990), Finding Groups in Data, John Wiley \& Sons.

Kunihama, T. and Dunson, D. B. (2016), "Nonparametric Bayes inference on conditional independence," Biometrika, 103, 35-47.

Kunihama, T., Herring, A., Halpern, C., and Dunson, D. (2016), "Nonparametric Bayes modeling with sample survey weights," Statistics and Probability Letters, 113, 41-48.

Madrigal, A. (2012), "Sorry, young man, you're not the most important demographic in tech," http://www.theatlantic.com/technology/archive/2012/06/sorry-youngman-youre-not-the-most-important-demographic-in-tech/258087.

Maimon, O. and Rokach, L. (2010), Data mining and knowledge discovery handbook, Springer.

Müller, P., Erkanli, A., and West, M. (1996), "Bayesian curve fitting using multivariate normal mixtures," Biometrika, 83, 67-79. 
Müller, P. and Mitra, R. (2013), "Bayesian nonparametric inference: Why and how?" Bayesian Analysis, 8, 269-302.

Müller, P. and Quintana, F. (2004), "Nonparametric Bayesian data analysis," Statistical Science, 19, 95-110.

Murray, J. and Reiter, J. (2016), "Multiple imputation of missing categorical and continuous values via Bayesian mixture models with local dependence," Journal of the American Statistical Association.

Norets, A. and Pelenis, J. (2012), "Bayesian modeling of joint and conditional distributions," Journal of Econometrics, 168, 332-346.

Paiva, T. and Reiter, J. P. (2015), "Stop or continue data collection: A nonignorable missing data approach for continuous variables," arXiv:1511.02189, Stat.ME.

Papageorgiou, G., Richardson, S., and Best, N. (2015), "Bayesian nonparametric models for spatially indexed data of mixed type," Journal of the Royal Statistical Society, 77, 973-999.

Peng, H., Long, F., and Ding, C. (2005), "Feature selection based on mutual information: criteria of max-dependency, max-relevance, and min-redundancy," IEEE Transactions on Pattern Analysis and Machine Intelligence, 27, 1226-1238.

Rainie, L., Zickuhr, K., Purcell, K., Madden, M., and Brenner, J. (2012), "The rise of ereading," http://libraries .pewinternet.org/2012/04/04/part-2-the-generalreading-habits-of-americans.

Rässler, S. (2004), "Data fusion: Identification problems, validity, and multiple imputation," Austrian Journal of Statistics, 33, 153-171.

Rubin, D. (1987), Multiple Imputation for Nonresponse in Surveys, New York: John Wiley and Sons.

Savitsky, T., Toth, D., and Sverchov, M. (2016), "Bayesian estimation under informative sampling," Electronic Journal of Statistics, To appear. 
Schifeling, T. and Reiter, J. (2016), "Incorporating marginal prior information in latent class models," Bayesian Analysis, 11, 499-518.

Shahbaba, B. and Neal, R. (2009), "Nonlinear modeling using Dirichlet process mixtures," Journal of Machine Learning Research, 10, 1829-1850.

Si, Y., Pillai, N., and Gelman, A. (2015), "Bayesian nonparametric weighted sampling inference," Bayesian Analysis, 10, 605-625.

Siddiqi, N. (2006), Credit risk scorecards: Developing and implementing intelligent credit scoring, John Wiley \& Sons.

van der Putten, P., Kok, J. N., and Gupta, A. (2002), "Data fusion through statistical matching," Working paper 4342-02, MIT Sloan School of Management.

Wade, S., Dunson, D., Perone, S., and Trippa, L. (2014), "Improving prediction from Dirichlet process mixtures via enrichment," Journal of Machine Learning Research, 15, 1041-1071.

Wicken, G. and Elms, S. (2009), "Demystifying data fusion - the "why?", the "how?" and the "wow!"," Tech. rep., Advertising Research Foundation Week of Workshops, New York.

\section{A Appendix}

\section{A.1 Prior Specification}

The particular matrix-normal base distribution for the regression coefficient matrices $\boldsymbol{\beta}_{h}$ implies that elements of $\boldsymbol{\beta}_{h}$ in the same row have the same variance, but the variance differs for elements in different columns. This is a reasonable assumption because the variables in $\mathbf{Z}^{(R)}$ are standardized, and the cut-offs $\gamma_{j, 1}$ and $\gamma_{j, k_{j}^{(R)}-1}$ for $j=1, \ldots, p_{o}$ can be fixed to values that imply all variables in $\left(\mathbf{W}^{(R)}, \mathbf{Z}^{(R)}\right)$ have the same scale. However, we want to allow the variances of regression coefficients to differ across covariates, hence the use of the $\boldsymbol{\tau}=\left(\tau_{1}^{2}, \ldots, \tau_{k}^{2}\right)$ vector. 
We assume a conjugate gamma distribution for the precision parameter $\alpha$. The number of effective global components in the mixture is influenced by $\alpha$. Thus, the shape and rate parameters can be fixed to small values, such as 0.5 , to represent a relatively noninformative prior on the number of global mixture components. We recommend fixing $a_{1}=\cdots=a_{d_{j}}=1$ in the Dirichlet base distribution so that the probability vectors $\boldsymbol{\psi}_{h}^{(j)}$ can encompass a wide variety of values.

To specify priors for the remaining parameters, consider the limiting case of the mixture as $\alpha \rightarrow 0^{+}$, which results in a single multivariate normal distribution for $\left(\mathbf{W}^{(R)}, \mathbf{Z}^{(R)}\right)$. Our objective is to center and scale the mixture kernel appropriately. Since the $\mathbf{Z}^{(R)}$ are standardized, fixing $\gamma_{j, 1} \approx-3$ and $\gamma_{j, k_{j}-1} \approx 3$ places each $\mathbf{Z}_{j}^{(R)}$ and $\mathbf{W}_{j}^{(R)}$ on similar scales. We use a conjugate matrix-normal prior for $\boldsymbol{\beta}_{0}$, assuming $\boldsymbol{\beta}_{0} \sim \operatorname{MN}(\mathbf{0}, \boldsymbol{I}, h \boldsymbol{I})$. This represents prior information that the regression coefficients are centered at zero. We assume $\tau_{j}^{2} \sim$ $\operatorname{IG}\left(a_{\tau}, b_{\tau}\right)$, possibly truncated to the lie below a finite value (e.g., 6) if numerical problems arise, and $\boldsymbol{S} \sim \mathrm{W}\left(a_{S}, \boldsymbol{B}_{S}\right)$. Let $v_{j}$ denote an estimate for the variance of $\left(\boldsymbol{W}^{(R)}, \boldsymbol{Z}^{(R)}\right)_{j}$, which is given by $\left(\left(\gamma_{j, k_{j}^{(R)}-1}-\gamma_{j, 1}\right) / 4\right)^{2}$, for ordinal variables $j=1, \ldots, p_{o}$. For continuous variables $j=p_{o}+1, \ldots, p_{o}+p_{c}$, the quantity $\left(\operatorname{range}\left(Z_{j-p_{o}}^{(R)}\right) / 4\right)^{2}$ can be used as a proxy for variance. Under the standardization and cut-off points described, each $v_{j} \approx 1.5^{2}$. The marginal prior variance for $\left(\boldsymbol{W}^{(R)}, \boldsymbol{Z}^{(R)}\right)_{j} \mid \boldsymbol{D}$ is $a_{S}\left(\boldsymbol{B}_{S}\right)_{j j}\left(\nu-p_{c}-p_{o}-1\right)^{-1}+\left(b_{\tau}\left(a_{\tau}-1\right)^{-1}+h\right) \sum_{l=1}^{k} D_{l}^{2}$. For a default prior, we fix $a_{\tau}, a_{S}$, and $\nu$ to relatively small values. Because the design vector $\boldsymbol{D}$ consists of a one for the intercept term followed by only zeroes and ones, the smallest value it can take is 1 . To be conservative, we fix $\sum_{l=1}^{K} D_{l}^{2}$ to 1 , and determine values of $\boldsymbol{B}_{S}$, $b_{\tau}$, and $h$ such that marginal prior variance is approximately equal to $v$. For instance, we can set $\boldsymbol{B}_{s}=\left(\nu-p_{c}-p_{o}-1\right)\left(3 a_{S}\right)^{-1} \operatorname{diag}(v, \ldots, v), b_{\tau}=3^{-1}\left(a_{\tau}-1\right) v$, and $h=3^{-1} v$. This implies a prior variance for each element of $\boldsymbol{\beta}_{h}$ that allows a typical value of $Y_{j}^{(R)}$ to be as small as 1 or as large as $k_{j}^{(R)}$. It also centers each diagonal element of $\boldsymbol{\Sigma}_{h}$ at 0.75 , which is reasonable given that each $W_{j}^{(R)}$ and $Z_{j}^{(R)}$ range from around -3 to 3 . Although this can be used as a default approach to prior specification, in our experience inferences like density estimates and regression functionals are insensitive to the choice of values for the parameters 
of these hyperpriors.

\section{A.2 Algorithm for Selecting Variables in $d(\cdot, \cdot)$}

We select variables in $\mathcal{F}$ in accordance with the mutual information of each $\mathcal{F}_{l}$, where $l=$ $1, \ldots, q$, with $\mathbf{X}^{(R)}$, as well as the mutual information between pairs of $\left(\mathcal{F}_{l}, \mathcal{F}_{l^{\prime}}\right), l \neq l^{\prime}$. Ding and Peng (2003) and Peng et al. (2005) provide an algorithm referred to as minimalredundancy-maximal-relevance (mRMR) which we adapt for our purposes, extending their algorithm to handle multivariate $\mathbf{X}^{(R)}$. The algorithm proceeds as follows.

1. Set $\boldsymbol{S}=\{\emptyset\}$ and $\boldsymbol{G}=\{1, \ldots, q\}$. Calculate empirical estimates of $I\left(\mathcal{F}_{l}, X_{j}^{(R)}\right)$, for $l=1, \ldots, q$ and $j=1, \ldots, p_{n}$, and then $I_{\mathcal{F}_{l}, x}^{\max }$, for $l=1, \ldots, q$. Denote these estimates by $\hat{I}\left(\mathcal{F}_{l}, X_{j}^{(R)}\right)$ and $\hat{I}_{\mathcal{F}_{l}, x}^{\max }$.

2. Find $l^{*}=\operatorname{argmax}_{l \in \boldsymbol{G}}\left\{\hat{I}_{\mathcal{F}_{l}, x}^{\max }\right\}$. Set $\boldsymbol{S}=\mathcal{F}_{l^{*}}$, and $\boldsymbol{G}=\{1, \ldots, q\} \backslash l^{*}$. Check whether or not to stop adding variables using some stopping criterion.

3. Repeat the following steps until the stopping criterion is satisfied.

(a) Find $l^{*}=\operatorname{argmax}_{l \in G}\left\{\hat{I}_{\mathcal{F}_{l}, x}^{\max }-|\boldsymbol{S}|^{-1} \sum_{j \in S} I\left(\mathcal{F}_{l}, \mathcal{F}_{j}\right)\right\}$.

(b) Add $\mathcal{F}_{l^{*}}$ to the set $\boldsymbol{S}$ and remove $l^{*}$ from $\boldsymbol{G}$.

The selected subset of variables $\mathcal{F}_{\boldsymbol{S}}$ are assigned $w_{j}=1 /|\boldsymbol{S}|$ in the distance function, and all others are assigned $w_{j}=0$. To estimate mutual information $I\left(\mathcal{F}_{l}, X_{j}^{(R)}\right)$ for discrete $\mathcal{F}_{l}$, we use the empirical discrete distribution of $\left(\mathcal{F}_{l}, X_{j}^{(R)}\right)$ in the calculation. When $\mathcal{F}_{l}$ is continuous, a simple and effective method of estimating the mutual information involves discretizing $\mathcal{F}_{l}$ (Ding and Peng, 2003). The marginal distribution for $\mathbf{X}^{(R)}$ depends on only the variables in $\mathcal{F}$ that are assigned non-zero weight, so one should include all variables that are moderately predictive of $\mathbf{X}^{(R)}$.

Related feature selection algorithms (Battiti, 1994 , Ding and Peng, 2003; Eztévez et al. 2009) stop when a pre-specified number of features have been chosen, and use cross validation to determine the optimal number of features. This is impractical and inefficient for our 
purposes, as the variable selection problem is not the primary inferential or modeling focus. Rather, it is a component of a complex joint model that is introduced to allow for further dependence of $\mathcal{R}$, and especially $\mathbf{X}^{(R)}$, on $\mathcal{F}$.

Instead, we make use of measures of redundancy and relevancy. We propose to stop selecting variables when none remain that explain a significant proportion of the uncertainty in any $\mathbf{X}^{(R)}$ variable, or when each remaining $\mathcal{F}$ variable to choose from is already well explained by the chosen set $\mathcal{F}_{\boldsymbol{S}}=\left\{\mathcal{F}_{s}: s \in \boldsymbol{S}\right\}$, where each $s_{j} \in\{1, \ldots, q\}, j=1, \ldots,|\boldsymbol{S}|$.

For generic random variable $A$, let $H(A)$ be the entropy of $A$. Define a normalized version of mutual information, $I^{*}\left(\mathcal{F}_{l} ; X_{j}^{(R)}\right)=I\left(\mathcal{F}_{l}, X_{j}^{(R)}\right) / H\left(X_{j}^{(R)}\right)$, as the proportion of uncertainty in $X_{j}^{(R)}$ that is explained by $\mathcal{F}_{l}$. We use this quantity to determine whether or not $\mathcal{F}_{l}$ is relevant in explaining $X_{j}^{(R)}$. Also, let $I^{*}\left(\mathcal{F}_{l} ; \mathcal{F}_{l^{\prime}}\right)=I\left(\mathcal{F}_{l}, \mathcal{F}_{l^{\prime}}\right) / H\left(\mathcal{F}_{l^{\prime}}\right)$, for $l, l^{\prime} \in 1, \ldots, q$ be the proportion of uncertainty in $\mathcal{F}_{l^{\prime}}$ that is explained by $\mathcal{F}_{l}$. As a measure of redundancy between $\mathcal{F}_{l}$ and $\mathcal{F}_{\boldsymbol{S}}$, we use $\max _{j \in \boldsymbol{S}}\left\{I^{*}\left(\mathcal{F}_{s_{j}} ; \mathcal{F}_{l}\right)\right\}$. If one or more of the variables in $\mathcal{F}_{\boldsymbol{S}}$ already explains a large amount of the uncertainty in $\mathcal{F}_{l}$, then $\mathcal{F}_{l}$ is considered redundant.

We express the relevancy stopping condition as $\max _{l \in G}\left\{\max \left\{I^{*}\left(\mathcal{F}_{l}, X_{1}^{(R)}\right), \ldots, I^{*}\left(\mathcal{F}_{l}, X_{q}^{(R)}\right)\right\}\right\}<$ $t_{1}$, and the redundancy stopping condition as $\min _{l \in G}\left\{\max _{j \in \boldsymbol{S}}\left\{I^{*}\left(\mathcal{F}_{s_{j}} ; \mathcal{F}_{l}\right)\right\}\right\}>t_{2}$. Here, $t_{1}$ and $t_{2}$ are user defined thresholds between 0 and 1 . A reasonable value for $t_{2}$ is around 0.7 to 0.9 , and a reasonable value for $t_{1}$ is closer to 0 , such as 0.05 or 0.1 . Generally, information values below 0.02 indicate a variable is not predictive, values 0.02 to 0.1 are considered weakly predictive, and values above 0.1 are considered moderately to strongly predictive Siddiqi, 2006). The larger $t_{1}$ is and the smaller $t_{2}$ is, the more parsimonious the selected variable set is. Generally, the number of variables one uses will depend on what the variables are (in applications with knowledge about the existence of relationships between the $\mathcal{F}$ and $\mathbf{X}^{(R)}$ ) and the importance of computational efficiency, as the more variables that are selected, the more time it takes the MCMC algorithm to run. 


\section{A.3 Posterior Inference: Full Conditionals}

\section{The Mixing Parameters}

The full conditionals for the mixing parameters $\left\{\boldsymbol{\theta}_{h}=\left(\boldsymbol{\beta}_{h}, \boldsymbol{\Sigma}_{h}, \boldsymbol{\psi}_{h}\right)\right\}$ arise by combining the likelihood terms in (1) and the base distributions in (3), as is standard in DP mixture models. Let $M_{h}=\left|\left\{H_{i}=h\right\}\right|$, or the size of cluster $h$. The full conditional for $\boldsymbol{\beta}_{h}$ is matrix-normal, or $\operatorname{vec}\left(\boldsymbol{\beta}_{h}^{T}\right)$ is multivariate normal. Let $\boldsymbol{T}=\operatorname{diag}\left(\tau_{1}^{2}, \ldots, \tau_{k}^{2}\right)$, and let $\boldsymbol{D}_{h}$ be an $M_{h}$ by $k$ matrix obtained by stacking the set of row vectors $\boldsymbol{D}\left(\mathbf{X}_{i}^{(R)}, \mathbf{Y}_{i}^{(F)}, \mathbf{Z}_{i}^{(F)}, \mathbf{X}_{i}^{(F)}\right)$, such that $H_{i}=h$. Let $\left(\mathbf{W}^{(R)}, \mathbf{Z}^{(R)}\right)_{h}$ be similarly defined. The multivariate normal full conditional has covariance matrix $\boldsymbol{V}_{\beta_{h}}=\left(\left(\boldsymbol{T} \otimes \boldsymbol{I}_{p_{o}+p_{c}}\right)^{-1}+\left(\boldsymbol{D}_{h}^{T} \boldsymbol{D}_{h}\right) \otimes \boldsymbol{\Sigma}_{h}^{-1}\right)^{-1}$, and mean vector $\boldsymbol{V}_{\beta_{h}}\left(\left(\boldsymbol{T} \otimes \boldsymbol{I}_{p_{o}+p_{c}}\right)^{-1} \operatorname{vec}\left(\boldsymbol{\beta}_{0}^{T}\right)+\left(\boldsymbol{D}_{h}^{T} \otimes \boldsymbol{\Sigma}_{h}^{-1}\right) \operatorname{vec}\left(\left(\mathbf{W}^{(R)}\right)_{h}^{T}\right)\right)$.

Alternatively, we can write the distribution for $\tilde{\mathbf{W}}_{i}^{(R)}=\left(\mathbf{W}_{i}^{(R)}, \mathbf{Z}_{i}^{(R)}\right)$ such that $H_{i}=h$ as $\left[\tilde{\mathbf{W}}_{i, j}^{(R)} \mid \tilde{\mathbf{W}}_{i,-j}^{(R)}\right]\left[\tilde{\mathbf{W}}_{i,-j}^{(R)}\right]$ for $j=1, \ldots, p_{o}+p_{c}$. This depends on the $j$ th column of $\boldsymbol{\beta}_{h}$, $\boldsymbol{\beta}_{h(\cdot, j)}$, only through the first univariate normal, which is $\mathrm{N}\left(\tilde{\mathbf{W}}_{i j}^{(R)} ; \boldsymbol{D}_{i} \boldsymbol{\beta}_{h(\cdot, j)}+\mu_{i, j}^{*}, \tilde{\boldsymbol{\Sigma}}_{h, j}\right)$ where $\mu_{i, j}^{*}=\boldsymbol{\Sigma}_{h(j,-j)}\left(\boldsymbol{\Sigma}_{h(-j,-j)}\right)^{-1}\left(\tilde{\mathbf{W}}_{i,-j}^{(R)}-\boldsymbol{D}_{i} \boldsymbol{\beta}_{h(\cdot,-j)}\right)$ and the conditional variance is $\tilde{\boldsymbol{\Sigma}}_{h, j}=$ $\boldsymbol{\Sigma}_{h(j, j)}-\boldsymbol{\Sigma}_{h(j,-j)}\left(\boldsymbol{\Sigma}_{h(-j,-j)}\right)^{-1} \boldsymbol{\Sigma}_{h(-j, j)}$. Here $\boldsymbol{D}_{i}=\boldsymbol{D}\left(\mathbf{X}_{i}^{(R)}, \mathbf{Y}_{i}^{(F)}, \mathbf{Z}_{i}^{(F)}, \mathbf{X}_{i}^{(F)}\right)$.

We therefore can update each column $j=1, \ldots, p_{o}+p_{c}$ of the matrix $\boldsymbol{\beta}_{h}$ from a multivariate normal with variance $\boldsymbol{V}_{h, j}=\left(\boldsymbol{T}^{-1}+\tilde{\boldsymbol{\Sigma}}_{h, j}^{-1}\left(\boldsymbol{D}_{h}^{T} \boldsymbol{D}_{h}\right)\right)^{-1}$ and mean $\boldsymbol{V}_{h, j}\left(\boldsymbol{T}^{-1} \boldsymbol{\beta}_{0 \cdot j}+\right.$ $\tilde{\Sigma}_{h, j}^{-1} \boldsymbol{D}_{h}^{T}\left(\left(\mathbf{W}^{(R)}\right)_{h(\cdot, j)}-\mu_{h, j}^{*}\right)$ where $\tilde{\Sigma}_{h, j}$ is the conditional variance of $\left(\tilde{\mathbf{W}}_{i j}^{(R)} \mid \tilde{\mathbf{W}}_{i,-j}^{(R)}, H_{i}=h\right)$ and $\mu_{h, j}^{*}$ is obtained by concatenating the set of $\mu_{i, j}^{*}$ over $\left\{i: H_{i}=h\right\}$.

When cluster $h$ is empty, i.e., $M_{h}=0$, we draw from the base distribution, which we can do by simulating $\operatorname{vec}\left(\boldsymbol{\beta}_{h}\right) \sim \mathrm{N}\left(\operatorname{vec}\left(\boldsymbol{\beta}_{0}\right), \boldsymbol{I}_{p_{o}+p_{c}} \otimes \boldsymbol{T}\right)$.

The covariance matrices $\boldsymbol{\Sigma}_{h}$ are updated from $\operatorname{IW}\left(\nu+M_{h}, \boldsymbol{S}+\sum_{\left\{i: H_{i}=h\right\}}\left(\tilde{\mathbf{W}}_{i}^{(R)}-\boldsymbol{\beta}_{h} \boldsymbol{D}_{i}\right)\left(\tilde{\mathbf{W}}_{i}^{(R)}-\right.\right.$ $\left.\left.\boldsymbol{\beta}_{h} \boldsymbol{D}_{i}\right)^{T}\right)$ where $\boldsymbol{D}_{i}=\boldsymbol{D}\left(\mathbf{X}_{i}^{(R)}, \mathbf{Y}_{i}^{(F)}, \mathbf{Z}_{i}^{(F)}, \mathbf{X}_{i}^{(F)}\right)$. If $M_{h}=0, \boldsymbol{\Sigma}_{h}$ is updated from the base distribution IW $(\nu, \boldsymbol{S})$. The probability vectors of the categorical distributions have full conditionals which are Dirichlet distributed: $\boldsymbol{\psi}_{h}^{(j)} \sim \operatorname{Dirichlet}\left(a_{1}+\sum_{\left\{i: H_{i}=h\right\}} 1\left(X_{i j}^{(R)}=1\right), \ldots, a_{d_{j}}+\right.$ $\left.\sum_{\left\{i: H_{i}=h\right\}} 1\left(X_{i j}^{(R)}=d_{j}\right)\right)$. Again, if $M_{h}=0$, then the update is $\operatorname{simply} \operatorname{Dirichlet}\left(a_{1}, \ldots, a_{d_{j}}\right)$.

We simulate from the posterior distribution of $\boldsymbol{\beta}_{h}, \boldsymbol{\Sigma}_{h}$, and $\boldsymbol{\psi}_{h}^{(j)}$, for $h=1, \ldots, N$, and $j=1, \ldots, p_{n}$ using the full conditionals given above. 


\section{The Mixture Allocation Variables and Local DP Parameters}

The mixture configuration variables $H_{i}$, where $i=1, \ldots, n$ are simulated from categorical distributions. We have

$$
p\left(H_{i} \mid \ldots\right) \propto \sum_{l=1}^{N\left(\boldsymbol{f}_{i}\right)} p_{l}{ }^{\prime}\left(\boldsymbol{f}_{i}\right) \delta_{\pi_{l}\left(\boldsymbol{f}_{i}\right)}(\cdot)
$$

, where $p_{l}{ }^{\prime}\left(\boldsymbol{f}_{i}\right) \propto p_{l}\left(\boldsymbol{f}_{i}\right) \mathrm{N}\left(\boldsymbol{\beta}_{\pi_{l}\left(\boldsymbol{f}_{i}\right)} \boldsymbol{D}\left(\mathbf{X}_{i}^{(R)}, \mathbf{Y}_{i}^{(F)}, \mathbf{Z}_{i}^{(F)}, \mathbf{X}_{i}^{(F)}\right), \boldsymbol{\Sigma}_{\pi_{l}\left(\boldsymbol{f}_{i}\right)}\right) \prod_{j=1}^{p_{n}} \psi_{\pi_{l}\left(\boldsymbol{f}_{i}\right), X_{i j}^{(R)}}^{(j)}$.

The full conditionals for $V_{h}, h=1, \ldots, N$, are derived as follows:

$$
\begin{aligned}
p\left(V_{h} \mid \ldots\right) & \propto \operatorname{beta}\left(V_{h} ; 1, \alpha\right) \prod_{i=1}^{n} \sum_{l=1}^{N\left(\boldsymbol{C}_{i}\right)} p_{l}\left(\boldsymbol{f}_{i}\right) \delta_{\pi_{l}\left(\boldsymbol{f}_{i}\right)}\left(H_{i}\right) \\
& \propto \operatorname{beta}\left(V_{h} ; 1, \alpha\right) \prod_{\substack{i: H_{i}=h \\
H_{i} \neq \pi_{N\left(c_{i}\right)}\left(c_{i}\right)}} V_{h} \prod_{\substack{i: H_{i}>h \\
h \in \eta_{\boldsymbol{f}_{i}}}}\left(1-V_{h}\right) \\
& \propto \operatorname{beta}\left(V_{h} ; 1+\sum_{i=1}^{n} 1\left(H_{i}=h, H_{i} \neq \pi_{N\left(\boldsymbol{f}_{i}\right)}\left(\boldsymbol{f}_{i}\right)\right), \alpha+\sum_{i=1}^{n} 1\left(H_{i}>h \text { and } h \in \eta_{\boldsymbol{f}_{i}}\right)\right) .
\end{aligned}
$$

The full conditional for $\boldsymbol{\Gamma}_{h}$ is

$$
p\left(\boldsymbol{\Gamma}_{h} \mid \ldots\right) \propto p\left(\boldsymbol{\Gamma}_{h}\right) \prod_{\left\{i: H_{i}=h\right\}} 1\left(d\left(\boldsymbol{f}_{i}, \boldsymbol{\Gamma}_{h}\right)<d^{*}\right) .
$$

The full conditional in general depends on the choice of distance function, which in CMM-Mix is $d\left(\boldsymbol{f}_{i}, \boldsymbol{\Gamma}_{h}\right)=\sum_{j=1}^{q} w_{j} d_{j}^{e}\left(f_{i j}, \Gamma_{h j}\right)$. The term $\sum_{j=1}^{q} w_{j} d_{j}^{e}\left(f_{i j}, \Gamma_{h j}\right)<d^{*}$ implies $a_{i l}<\Gamma_{h l}<b_{i l}$, for any $l=1, \ldots, q$. Combined with the product of independent uniforms prior for $\boldsymbol{\Gamma}_{h}$, the full conditionals for each $\Gamma_{h l}$ are uniform.

For $l=1, \ldots, q_{o}, \Gamma_{h l}$ corresponds to ordinal variable $\mathcal{F}_{l}$, with $\mathcal{F}_{l} \in\left\{1, \ldots, k_{l}^{(F)}\right\}$, and $d\left(\mathcal{F}_{i}, \Gamma_{h}\right)<d^{*}$ implies $a_{i l}<\Gamma_{h l}<b_{i l}$ where $a_{i l}=\mathcal{F}_{i l}+\left(\left(k_{l}^{(F)}-1\right) / w_{l}\right)\left(d^{*}-\sum_{j \neq l} w_{j} d_{j}^{e}\left(\mathcal{F}_{i j}, \Gamma_{h j}\right)\right)$ and $b_{i l}=\mathcal{F}_{i l}-\left(\left(k_{l}^{(F)}-1\right) / w_{l}\right)\left(d^{*}-\sum_{j \neq l} w_{j} d_{j}^{e}\left(\mathcal{F}_{i j}, \Gamma_{h j}\right)\right)$. Therefore the full conditional for $\Gamma_{h l}$ is uniform, with lower bound equal to the smallest value in $\left\{1, \ldots, k_{l}^{(F)}\right\}$ that is greater than $\max _{\left\{i: H_{i}=h\right\}}\left\{a_{i l}\right\}$, and upper bound equal to the largest value in $\left\{1, \ldots, k_{l}^{(F)}\right\}$ that is less than $\min _{\left\{i: H_{i}=h\right\}}\left\{b_{i l}\right\}$. For $\left\{l: w_{l}=0\right\}, \Gamma_{h l}$ is simulated from the prior, which is uniform on $\left\{1, \ldots, k_{l}^{(F)}\right\}$.

For $l=q_{o}+1, \ldots, q_{o}+q_{c}, \Gamma_{h l}$ corresponds to continuous variable $\mathcal{F}_{l}$, and under a uniform $\left(a_{l}^{\Gamma}, b_{l}^{\Gamma}\right)$ prior for $\Gamma_{h l}, d\left(\mathcal{F}_{i}, \Gamma_{h}\right)<d^{*}$ implies $a_{i l}<\Gamma_{h l}<b_{i l}$ where $a_{i l}=\mathcal{F}_{i l}+$ 
$\left(\max \left(\mathcal{F}_{l}\right)-\min \left(\mathcal{F}_{l}\right)\right)\left(d^{*}-\sum_{j \neq l} w_{j} d_{j}^{e}\left(\mathcal{F}_{i j}, \Gamma_{h j}\right)\right) / w_{l}$ and $b_{i l}=\mathcal{F}_{i l}-\left(\max \left(\mathcal{F}_{l}\right)-\min \left(\mathcal{F}_{l}\right)\right)\left(d^{*}-\right.$ $\left.\sum_{j \neq l} w_{j} d_{j}^{e}\left(\mathcal{F}_{i j}, \Gamma_{h j}\right)\right) / w_{l}$. Therefore the full conditional for $\Gamma_{h l}$ is uniform, with lower bound equal to $\max \left(\max _{\left\{i: H_{i}=h\right\}}\left\{a_{i l}\right\}, a_{l}^{\Gamma}\right)$, and upper bound equal to $\min \left(\min _{\left\{i: H_{i}=h\right\}}\left\{b_{i l}\right\}, b_{l}^{\Gamma}\right)$. For $\left\{l: w_{l}=0\right\}, \Gamma_{h l}$ is simulated from the prior, which is uniform $\left(a_{l}^{\Gamma}, b_{l}^{\Gamma}\right)$.

For $l=q_{o}+q_{c}+1, \ldots, q, \Gamma_{h l}$ corresponds to nominal variable $\mathcal{F}_{l} \in\left\{1, \ldots, d_{l}^{(F)}\right\}$, and $d\left(\mathcal{F}_{i}, \Gamma_{h}\right)<d^{*}$ implies

$$
1\left(\mathcal{F}_{i l} \neq \Gamma_{h l}\right)<b_{i l}=\left(d^{*}-\sum_{j \neq l} w_{j} d_{j}^{e}\left(\mathcal{F}_{i j}, \Gamma_{h j}\right)\right) / w_{l}
$$

and therefore if $\min _{\left\{i: H_{i}=h\right\}}\left(b_{i l}\right)<1$, this implies a degenerate full conditional for $\Gamma_{h k}$ at $\mathcal{F}_{i l}$. Otherwise, $\Gamma_{h l}$ is drawn randomly from $\left\{1, \ldots, d_{l}^{(F)}\right\}$. For $\left\{k: w_{k}=0\right\}, \Gamma_{h l}$ is simulated from the prior, which is uniform on $\left\{1, \ldots, d_{l}^{(F)}\right\}$.

\section{The Base Distribution Hyperparameters}

The full conditional for $\alpha$ is the same as under the standard DP applied with truncation. Under the prior $\alpha \sim \operatorname{gamma}\left(a_{\alpha}, b_{\alpha}\right)$, then the posterior for $\alpha$ is proportional to gamma $\left(a_{\alpha}+\right.$ $\left.N, b_{\alpha}-\sum_{h=1}^{N} \log \left(1-V_{h}\right)\right)$.

Under the prior $\boldsymbol{\beta}_{0} \sim \mathrm{MN}_{k \times\left(p_{o}+p_{c}\right)}(\mathbf{0}, \boldsymbol{I}, h \boldsymbol{I})$, the full conditional for each $\boldsymbol{\beta}_{0 m r}, m=$ $1, \ldots, k, r=1, \ldots, p_{o}+p_{c}$ is then $\mathrm{N}\left(\left(h^{-1}+N \tau_{m}^{-2}\right)^{-1}\left(\tau_{m}^{-2} \sum_{l=1}^{N} \beta_{l(m, r)}\right),\left(h^{-1}+N \tau_{m}^{-2}\right)^{-1}\right)$.

Assuming $\tau_{j}^{2} \sim \operatorname{IG}\left(a_{\tau}, b_{\tau}\right)$ for $j=1, \ldots, k$, we obtain a posterior full conditional for $\tau_{j}^{2}$ which is $\operatorname{IG}\left(a_{\tau}+0.5 N\left(p_{o}+p_{c}\right), b_{\tau}+0.5 \sum_{h=1}^{N} \sum_{j=1}^{p_{o}+p_{c}}\left(\beta_{h(l, j)}-\beta_{0(l, j)}\right)^{2}\right)$.

With a prior $\boldsymbol{S} \sim \operatorname{Wish}\left(a_{S}, \boldsymbol{B}_{S}\right), \boldsymbol{S}$ is sampled from $\operatorname{Wish}\left(N \nu+a_{S},\left(\boldsymbol{B}_{S}^{-1}+\sum_{h=1}^{N} \boldsymbol{\Sigma}_{h}^{-1}\right)^{-1}\right)$.

\section{Latent Continuous Variables and Missing Data Imputation}

The latent continuous random variables $W_{i j}^{(R)}, i=1, \ldots, n$, and $j=1, \ldots, p_{o}$ have full conditionals $p\left(W_{i j}^{(R)} \mid \ldots\right) \propto \mathrm{N}\left(W_{i j}^{(R)} ; \tilde{\mu}_{i j}, \tilde{\sigma}_{i j}^{2}\right)$ where $\tilde{\mu}_{i j}$ and $\tilde{\sigma}_{i j}^{2}$ are the conditional normal mean and variance for $W_{i j}^{(R)}$ resulting from the multivariate normal distribution $\mathrm{N}\left(\mathbf{W}_{i}^{(R)}, \mathbf{Z}_{i}^{(R)} ; \boldsymbol{D}\left(\mathbf{X}_{i}^{(R)}, \mathbf{Y}_{i}^{(F)}, \mathbf{Z}_{i}^{(F)}, \mathbf{X}_{i}^{(F)}\right) \boldsymbol{\beta}_{H_{i}}, \boldsymbol{\Sigma}_{H_{i}}\right)$. If $Y_{i j}^{(R)}$ is observed, then $W_{i j}^{(R)}$ must lie 
in the interval $\left(\gamma_{j, Y_{i j}^{(R)}-1}, \gamma_{j, Y_{i j}^{(R)}}\right]$, i.e., its full conditional is truncated normal. If $Y_{i j}^{(R)}$ is missing, then $W_{i j}^{(R)}$ is sampled from the normal distribution without any truncation.

Similarly, missing $Z_{i j}^{(R)}$ are simulated from the normal distribution $\mathrm{N}\left(Z_{i j}^{(R)} ; \tilde{\mu}_{i j}, \tilde{\sigma}_{i j}^{2}\right)$ where $\tilde{\mu}_{i j}$ and $\tilde{\sigma}_{i j}^{2}$ are the conditional normal mean and variance for $Z_{i j}^{(R)}$ resulting from the multivariate normal $\mathrm{N}\left(\left(\mathbf{W}_{i}^{(R)}, \mathbf{Z}_{i}^{(R)}\right) ; \boldsymbol{D}\left(\mathbf{X}_{i}^{(R)}, \mathbf{Y}_{i}^{(F)}, \mathbf{Z}_{i}^{(F)}, \mathbf{X}_{i}^{(F)}\right) \boldsymbol{\beta}_{H_{i}}, \boldsymbol{\Sigma}_{H_{i}}\right)$.

In order to improve mixing, we can block update the $W_{i j}^{(R)}$ and $Z_{i j}^{(R)}$ using the fact that $p\left(\tilde{\mathbf{W}}_{i}^{(R)} \mid\right.$ data, $\left.\theta\right)=p\left(\tilde{W}_{i 1}^{(R)} \mid\right.$ data, $\left.\theta\right) \prod_{j=2}^{p_{o}+p_{c}} p\left(\tilde{W}_{i j}^{(R)} \mid\left(\tilde{W}_{i 1}^{(R)}, \ldots, \tilde{W}_{i, j-1}^{(R)}\right)\right.$, data, $\left.\theta\right)$, where $\theta$ indicates all model parameters. Letting $\boldsymbol{D}_{i}=\boldsymbol{D}\left(\mathbf{X}_{i}^{(R)}, \mathbf{Y}_{i}^{(F)}, \mathbf{Z}_{i}^{(F)}, \mathbf{X}_{i}^{(F)}\right)$, we sample $\tilde{W}_{i 1}^{(R)} \sim \mathrm{N}\left(\tilde{W}_{i 1}^{(R)} ; \boldsymbol{D}_{i} \boldsymbol{\beta}_{H_{i}(\cdot, 1)},\left(\Sigma_{H_{i}}\right)_{11}\right)$, with truncation to $\left(\gamma_{1, Y_{i 1}^{(R)}-1}, \gamma_{1, Y_{i 1}^{(R)}}\right]$ if $Y_{i 1}^{(R)}$ is observed. For $j=2, \ldots, p_{o}+p_{c}$, let $\mu_{i j}^{*}$ and $\left(\sigma^{*}\right)_{i j}^{2}$ denote the conditional mean and variance for $\left(\tilde{W}_{i j}^{(R)} \mid \tilde{W}_{i 1}^{(R)}, \ldots, \tilde{W}_{i, j-1}^{(R)}\right)$, and simulate $\tilde{W}_{i j}^{(R)} \sim \mathrm{N}\left(\tilde{W}_{i j}^{(R)} ; \mu_{i j}^{*},\left(\sigma^{*}\right)_{i j}^{2}\right)$, with truncation to $\left(\gamma_{j, Y_{i j}^{(R)}-1}, \gamma_{j, Y_{i j}^{(R)}}\right]$ for $j=2, \ldots, p_{o}$ if $Y_{i j}^{(R)}$ is observed.

Missing $X_{i j}^{(R)}$ are simulated from categorical distributions on $\left\{1, \ldots, d_{j}^{(R)}\right\}$ with probabilities proportional to

$$
\left(\psi_{H_{i}, 1}^{(j)} \mathrm{N}\left(\mathbf{W}_{i}^{(R)}, \mathbf{Z}_{i}^{(R)} ; \boldsymbol{D}_{1} \boldsymbol{\beta}_{H_{i}}, \boldsymbol{\Sigma}_{H_{i}}\right), \ldots, \psi_{H_{i}, d_{j}^{(R)}}^{(j)} \mathrm{N}\left(\mathbf{W}_{i}^{(R)}, \mathbf{Z}_{i}^{(R)} ; \boldsymbol{D}_{d_{j}^{(R)}} \boldsymbol{\beta}_{H_{i}}, \boldsymbol{\Sigma}_{H_{i}}\right)\right)
$$

where $\boldsymbol{D}_{l}$ indicates the current value of $\boldsymbol{D}\left(\mathbf{X}_{i}^{(R)}, \mathbf{Y}_{i}^{(F)}, \mathbf{Z}_{i}^{(F)}, \mathbf{X}_{i}^{(F)}\right)$ in which $X_{i j}^{(R)}=l$. 